\title{
STATIONARY MEASURES FOR THE FLOW OF A LINEAR DIFFERENTIAL EQUATION DRIVEN BY WHITE NOISE
}

\author{
BY \\ HARRY DYM(1)
}

1. Introduction. In this paper we study the stochastic process which is the trajectory (flow) in phase space ( $E^{n}, n$ dimensional Euclidean space) of the (formal) stochastic differential equation

$$
P(D) y_{1}=\left(D^{n}-a_{1} D^{n-1}-\cdots-a_{n}\right) y_{1}(t, \omega)=b_{n}(t, \omega), \quad t \geqq 0,
$$

with initial conditions

$$
y_{1}(0, \omega)=c_{1}, \quad D y_{1}(0, \omega)=c_{2}, \cdots, D^{n-1} y_{1}(0, \omega)=c_{n} .
$$

Here, as usual, $D$ denotes the operation of differentiation with respect to the variable $t ; t$ may be thought of as nonnegative time; the $a_{i}, i=1, \cdots, n$, are constants; the variable $\omega$ denotes a continuous sample path of the Brownian motion process $b_{n}(t, \omega)$ (i.e. $\omega$ maps $[0, \infty)$ into $E^{1}$ by sending $t \rightarrow \omega(t)=b_{n}(t, \omega)$ ); and $b_{n}(t, \omega)$ denotes the formal time derivative of $b_{n}(t, \omega)$. The process $b_{n}(t, \omega)$ is often referred to as white noise. Since the Brownian sample paths are, with probability one, nowhere differentiable the symbol $b_{n}(t, \omega)$ has no meaning within the framework of conventional (nongeneralized) functions. Nevertheless the (stochastic) integral

$$
\int_{0}^{t} f(s) b_{n}(s, \omega) d s=\int_{0}^{t} f(s) d b_{n}(s)
$$

has a meaningful interpretation for every square-integrable function $f$. Consequently the formal solution of (1.1) expressed in terms of a Green's function is a well-defined stochastic process which is in fact $(n-1)$ times differentiable. Hence the trajectory process which we shall study here is a meaningful process

Received by the editors May 18, 1965.

(1) This paper was written at M.I.T. as a Ph.D. thesis. The author wishes to thank Professor H. P. McKean Jr., his thesis supervisor, for contributing generously of his time and energy while guiding the research discussed herein. Both his technical assistance and his unflagging enthusiasm and interest proved invaluable. The author also wishes to thank Professor D. B. Ray for critically reading an early version of this paper and suggesting improvements. 
which could have been defined abstractly without reference to a differential equation. It is convenient nevertheless to think of this process as being generated by a differential equation.

REMARK 1.1. If $f$ is continuously differentiable, which case is adequate for our purposes, we may define

$$
\begin{aligned}
\int_{r}^{t} f(s) b_{n}(s, \omega) d s= & f(t) b_{n}(t, \omega)-f(r) b_{n}(r, \omega) \\
& -\int_{r}^{t} f(s) b_{n}(s, \omega) d s .
\end{aligned}
$$

Note that the right-hand side may be obtained by formally integrating the left-hand side by parts. It is subsequently not hard to verify that integrals over disjoint intervals are independent Gaussian random variables with zero mean and variance

$$
E\left(\int_{r}^{t} f(s) b_{n}(s, \omega) d s\right)^{2}=\int_{r}^{t} f^{2}(s) d s
$$

In the following section we shall show that the trajectory process $y$ is a Gaussian diffusion; a diffusion is a strong Markov process with continuous sample paths. Additional properties of the $y$ process are enumerated and discussed in $\S 3$. In $\$ 4$, drawing partially on the results of Khasminskii [14], we characterize transience and recurrence for a general class of diffusion processes in terms of the average amount of time such processes spend in bounded open sets (average sojourn time). Since the transition probabilities of the $y$ process are known explicitly it is possible to estimate the average sojourn time in a set and hence, using the characterization developed in $\S 4$, to establish conditions under which the $y$ process is transient or recurrent. Such estimates are carried out in $\$ 6$; in $\$ 5$ properties of the matrices $e^{A t}$ and $R(t)$ pertinent to such estimates are summarized. The main result of $\S 6$, Theorem 6.2 , may be phrased in the following fashion:

The trajectory process generated by the differential operator $P(D)$ will be recurrent if and only if $P(D)$ can be expressed in one of the five forms:

$$
D, \quad D^{2}+\beta^{2}, \quad P_{1}(D), \quad D P_{1}(D), \quad\left(D^{2}+\beta^{2}\right) P_{1}(D),
$$

where $P_{1}(z)$ is a polynomial with real coefficients all of whose roots lie in $\Lambda$, the open left half plane, and where $\beta>0$. A differential operator of any other form generates a transient process.

Note that the last statement, immediately above, is not redundant since in general a nonrecurrent process is not a transient process.

It is also shown that the trajectory process is transient or recurrent according as 
$\int_{1}^{\infty}|R(t)|^{-1 / 2} d t$ converges or diverges, where $|R(t)|$ denotes the determinant of the covariance matrix $R(t)$ for the process.

In $\$ 7$ we discuss the existence and uniqueness of invariant densities for the trajectory process. We show that every invariant density is a real analytic function on $E^{n}$ and is a solution of the differential equation $G^{*} f=0$ (Theorem 7.2). Conversely, subject to some growth restrictions, every nonnegative solution of $G^{*} f=0$ is an invariant density (Theorem 7.3). Let $P(D)=D^{n}-a_{1} D^{n-1}-\cdots-a_{n}$. The corresponding trajectory process has a particularly interesting invariant density, $\psi_{0}(u)=\exp \left(-\Sigma C_{i j} u_{i} u_{j}\right)$ where

$$
\begin{aligned}
& C_{i j}=a_{n+1-i} a_{n-j}-C_{i-1, j+1} \quad \text { if } i+j \equiv 0(\bmod 2), \\
& \quad C_{i j}=0 \text { if } i+j \equiv 1(\bmod 2), \\
& a_{0}=-1, \quad C_{i j}=C_{j i}, \quad C_{0 i}=C_{i, n+1}=0, \quad i, j=1, \cdots, n .
\end{aligned}
$$

If the process is recurrent then $\psi_{0}$ is, up to a multiplicative constant, the only invariant density (Theorem 9.2).

We show (Theorem 7.5) that $\psi_{0}$ is integrable if and only if the roots of $P(z)$ lie in $\Lambda$ and hence deduce, as a corollary, a curious analogue of the Routh-Hurwitz criterion.

Subsequently, in $\S 8$, we show that $-\log \psi_{0}$ may be uniquely characterized as the form $\phi(u)=\Sigma C_{i j} u_{i} u_{j}+\sum B_{j} u_{j}, C_{i j}=C_{j i}$, which is

(1) invariant on the streamlines of the "force free oscillator"

$$
\left(D^{n}-a_{2} D^{n-2}-a_{4} D^{n-4}-\cdots\right) v_{1}=0
$$

and for which

(2) $C_{n, n-2 j}=-a_{2 j+1}, j=0,1, \cdots,[(n-1) / 2](=$ largest integer $\leqq(n-1) / 2)$. In $\$ 9$ we introduce the "backward" process and show how it leads to a second characterization of $\psi_{0}$.

We mention in passing a few papers which deal with topics related to this thesis. McKean [16] has given a detailed description of the winding about the origin of the trajectory process generated by $P(D)=D^{2}$. Potter [20] has studied the process generated by the nonlinear differential equation $D^{2} y_{1}-g\left(y_{1}\right)=b_{2}$. Kac [13] has characterized the mean square time between the zeros of the solution, $u_{1}$, to the differential equation $\left(D^{2}-a_{1} D-a_{2}\right) u_{1}=b_{2}, t>-\infty$. An up-to-date treatment of the problem of determining the mean time between zeros for a continuous stationary Gaussian process has, incidentally, been given by Ito [12].

Of somewhat older vintage, though still of interest, are papers by Ming Chen Wang and Uhlenbeck [18], Doob [7], [8], and (portions of) a review article by Chandrasekbar [4]. 
2. The trajectory process $y$ is a Gaussian diffusion. Introducing the notation

$$
\begin{array}{ll}
y=\left[\begin{array}{c}
y_{1} \\
\vdots \\
y_{n}
\end{array}\right], \quad c=\left[\begin{array}{c}
c_{1} \\
\vdots \\
c_{n}
\end{array}\right], \\
\dot{b}=\left[\begin{array}{c}
0 \\
\vdots \\
\vdots \\
0 \\
\dot{b}_{n}
\end{array}\right], \quad A=\left[\begin{array}{cccccc}
0 & 1 & 0 & \cdots & 0 & 0 \\
0 & 0 & 1 & \cdots & 0 & 0 \\
& & \cdot & & & \\
& & & & & \\
0 & 0 & & & 0 & 1 \\
a_{n} & a_{n-1} & & a_{2} & a_{1}
\end{array}\right]
\end{array}
$$

where $y_{i}=D y_{i-1}, i=2, \cdots, n$ we can transform (1.1) into the first order vector differential equation

$$
\begin{gathered}
D y(t, \omega)=A y(t, \omega)+\dot{b}(t, \omega), \quad t \geqq 0, \\
y(0, \omega)=c .
\end{gathered}
$$

The formal solution to (2.1)

$$
y(t, \omega)=\int_{0}^{t} e^{\Lambda(t-s)} \dot{b}(s, \omega) d s+e^{\Lambda t} c
$$

is a random process on $E^{n}$ with components which may, in accordance with Remark 1.1, be expressed as

$$
y_{i}(t, \omega)=\int_{0}^{t}\left(A e^{A(t-s)}\right)_{i n} b_{n}(s, \omega) d s+\left(e^{A t} c\right)_{i}, \quad i=1, \cdots, n-1,
$$

$$
y_{n}(t, \omega)=b_{n}(t, \omega)+\int_{0}^{t}\left(A e^{A(t-s)}\right)_{n n} b_{n}(s, \omega) d s+\left(e^{A t} c\right)_{n}
$$

Clearly, for almost every $\omega, y_{i}(\cdot, \omega)$ is continuous on $[0, \infty)$; in fact $y_{i}$ is differentiable and $D y_{i}=y_{i+1}, i=1, \cdots, n-1$.

An easy manipulation of (2.2) shows that if $h>0$ then

$$
y(t+h, \omega)=\int_{t}^{t+h} e^{A(t+h-s)} b(s, \omega) d s+e^{A h} y(t, \omega) .
$$

Since $b_{n}(t, \omega)$ is a process with independent increments the two summands on the right are independent. Hence the future evolution of the process $y(t+h)$, conditioned on the present $y(t)$, is independent of the past $y(s): s<t$. In short $y$ is a Markov process. 
Equations (2.3) imply that every linear combination of the random variables $y_{i}(t, \cdot)=y_{i}(t), i=1, \cdots, n$, is a Gaussian random variable. Hence the $y_{i}(t)$ are jointly Gaussian with mean

$$
\mu_{i}(t)=E\left[y_{i}(t)\right]=\left(e^{A t} y(0)\right)_{i}
$$

and covariance

$$
R_{i j}(t)=E\left[\left(y_{i}(t)-\mu_{i}(t)\right)\left(y_{j}(t)-\mu_{j}(t)\right)\right]=\int_{0}^{t}\left(e^{A s}\right)_{i n}\left(e^{A s}\right)_{j n} d s .
$$

The probability that the vector $y(t)$ will be found inside an incremental volume $d v$, conditional upon $y(0)=c$, is thus equal to

$$
\begin{aligned}
P_{c}(t, d v) & =p(t ; c, v) d v \\
& =(2 \pi)^{-n / 2}|R(t)|^{-1 / 2} \exp \left(-\left\langle v-e^{A t} c, R^{-1}(t)\left(v-e^{A t} c\right)\right\rangle / 2\right) d v
\end{aligned}
$$

where $R^{-1}(t)$ and $|R(t)|$ denote respectively the inverse and the determinant of the covariance matrix $R(t)$ and $\langle\cdot, \cdot\rangle$ denotes the standard inner product for $E^{n} . R(t)$ is a symmetric positive definite matrix for $t>0$ so $|R(t)|>0$ and $R^{-1}(t)$ exists for all $t>0$.

Remark 2.1. Let $g$ denote the Green function for the initial value problem

$$
\begin{gathered}
\left(D^{n}-a_{1} D^{n-1}-\cdots-a_{n}\right) p=h, \quad t \geqq 0, \\
p(0)=D p(0)=\cdots=D^{n-1} p(0)=0,
\end{gathered}
$$

i.e., $g$ is the unique function such that

$$
p(t)=\int_{0}^{t} g(t-s) h(s) d s .
$$

Then, as may for example be deduced from (2.2), for $t \geqq 0$

Consequently

$$
\left(e^{A t}\right)_{\text {in }}=D^{(i-1)} g(t)=g^{(i-1)}(t), \quad i=1, \cdots, n .
$$

$$
R_{i j}(t)=\int_{0}^{t} g^{(i-1)}(s) g^{(j-1)}(s) d s
$$

This formula, in conjunction with the fact $[2, \mathrm{p} .89]$ that

$$
g(0)=\cdots=g^{(n-2)}(0)=0, \quad g^{(n-1)}(0+)=1,
$$

will be useful in analyzing the behavior of $R$.

Continuing each entry in the covariance matrix, $R$, analytically to the complex plane and expanding in a Taylor series about zero we deduce, as a first application of Remark 2.1, 
LEMMA 2.1 .

$$
R_{i j}(t) \sim[(2 n+1-i-j)(n-i) !(n-j) !]^{-1} t^{(2 n+1-i-j)} \text { as } t \rightarrow 0 .
$$

Proof. By Remark 2.1.

$$
\frac{d^{m+1}}{d t^{m+1}} R_{i j}(0)=\sum_{v=0}^{m}\left(\begin{array}{c}
m \\
v
\end{array}\right) g^{(i-1+v)}(0+) g^{(j-1+m-v)}(0+) .
$$

Since $g^{(k)}(0)=0$ for $k<n-1$ the first nonzero term in the Taylor expansion of $R_{i j}$ occurs when $i-1+v=j-1+m-v=n-1$, i.e. when $m=2 n-i-j$ and $v=n-i$.

Direct application of the inequality

$$
|R(t)| \leqq R_{11}(t) \cdots R_{n n}(t)
$$

[1, Theorem 7, p. 63] leads to

Corollary 1. There exists a constant $c>0$ such that for all $0 \leqq t \leqq T$, $|R(t)| \leqq c t^{n^{2}}$.

Consider the semigroup of operators

$$
H_{t}: f \rightarrow \int f(b) p(t ; \cdot, b) d b, \quad t \geqq 0
$$

and the adjoint semigroup

$$
H_{t}^{*}: f \rightarrow \int f(a) p(t ; a, \cdot) d a, \quad t \geqq 0
$$

where $f$ is a Borel measurable function on $E^{n}$ which is either bounded, in which case we say $f \in \mathscr{B}\left(E^{n}\right)$, or nonnegative. We show

THEOREM 2.1. If $f \in \mathscr{B}\left(E^{n}\right)$ then

$$
g(t, a)=H_{t} f(a)=\int f(b) p(t ; a, b) d b
$$

is a real analytic function on $(0, \infty) \times E^{n}$.

Proof. Since $p(t ; a, b)$ is real analytic on $(0, \infty) \times E^{n} \times E^{n}$ we can, introducing the notation

$$
\begin{aligned}
\alpha & =\left(\alpha_{1}, \cdots, \alpha_{n}\right), & \beta & =\left(\beta_{1}, \cdots, \beta_{n}\right), \\
a^{\alpha} & =a_{1}^{\alpha_{1}} \cdots a_{n}^{\alpha_{n}}, & |\beta| & =\left|\beta_{1}\right|+\cdots+\left|\beta_{n}\right|,
\end{aligned}
$$

where each $\alpha_{i}$ and $\beta_{i}, i=1, \cdots, n$, is a nonnegative integer, express $p(t ; a, b)$ as a power series 


$$
p(t ; a, b)=\Sigma A_{i, \alpha, \beta}\left(t-t_{0}\right)^{t} a^{\alpha} b^{\beta}
$$

which converges absolutely and hence uniformly on every compact neighborhood of $\left(t_{0}, 0,0\right)$ which is contained in $(0, \infty) \times E^{n} \times E^{n}$. Defining

$$
g_{N}(t, a)=\int_{\|b\| \leqq N} f(b) p(t ; a, b) d b
$$

the order of summation and integration may be interchanged to yield

$$
g_{N}(t, a)=\Sigma A_{i, x, \beta}\left(t-t_{0}\right)^{i} a^{\alpha} \int_{\|b\| \leqq N} b^{\beta} f(b) d b .
$$

The right-hand side of the last expression converges absolutely on a compact neighborhood of $\left(t_{0}, 0\right)$ which is contained in $(0, \infty) \times E^{n}$. Consequently $g_{N}$ is a real analytic function on $(0, \infty) \times E^{n}$. Now

$$
\begin{aligned}
\left|g_{N}(t, u+i v)\right| & \leqq \int|f(b)| p(t ; u+i v, b) \mid d b \\
& =\exp \left(\left\langle e^{A t} v, R^{-1}(t) e^{A t} v\right\rangle\left(\frac{1}{2}\right)\right) \int|f(b)| p(t ; u, b) d b .
\end{aligned}
$$

The sequence of functions $g_{N}(t, \cdot)$ is thus uniformly bounded and analytic on a complex neighborhood of $a$. Consequently the pointwise limit $g(t, \cdot)$ must also be analytic on this complex neighborhood of $a$ and hence since $a$ was chosen arbitrarily $g(t, \cdot)$ must be analytic on $E^{n}$ for each $t>0$.

We now fix $a$ and show analogously that $g_{N}(r+i s, a)$ is uniformly bounded on a complex neighborhood of $t>0$. It clearly suffices to consider $|R(r+i s)|^{1 / 2} g_{N}(r+i s, a) \mid$. Our principal concern here is of course the behavior of the exponent of the transition probability density.

Since $R^{-1}(t)$ is analytic for $t>0$ there exists a number $M>0$ such that

$$
\left|\left\langle\eta+i \xi,\left(R^{-1}(r+i s)-R^{-1}(r)\right)(\eta+i \xi)\right\rangle\right| \leqq M\left(\|\eta\|^{2}+\|\xi\|^{2}\right)
$$

on a small complex neighborhood of $t$ for all $\eta, \xi \in E^{n}$. Consequently

$$
\begin{aligned}
\operatorname{Re}\left\langle\eta+i \xi, R^{-1}(r\right. & +i s)(\eta+i \xi)\rangle \\
& \geqq \operatorname{Re}\left\langle\eta+i \xi, R^{-1}(r)(\eta+i \xi)\right\rangle-M\left(\|\eta\|^{2}+\|\xi\|^{2}\right) \\
& =\left\langle\eta, R^{-1}(r) \eta\right\rangle-\left\langle\xi, R^{-1}(r) \xi\right\rangle-M\left(\|\eta\|^{2}+\|\xi\|^{2}\right)
\end{aligned}
$$

on this neighborhood. Identifying $\eta+i \xi$ with $b-e^{A r}(\cos A s) a-i e^{A r}(\sin A s) a$, it follows readily that the sequence of analytic functions $|R(\cdot)|^{1 / 2} g_{N}(\cdot, a)$ is uniformly bounded on the above neighborhood. Hence the pointwise limit $|R(\cdot)|^{1 / 2} g(\cdot, a)$ is also analytic on this neighborhood of $t$. This means that $g(\cdot, a)$ is analytic on $(0, \infty)$ for each $a \in E^{n}$ and thus by Hartog's theorem, Bochner and Martin [3, Theorem 4, p. 140], $g$ is analytic on $(0, \infty) \times E^{n}$. 
REMARK 2.2. An analogous theorem may be proved for the adjoint semigroup, i.e. if $f \in \mathscr{B}\left(E^{n}\right)$ then $H_{t}^{*} f(a)$ is real analytic on $(0, \infty) \times E^{n}$.

Theorem 2.1 implies especially that the class of bounded continuous functions on $E^{n}, C\left(E^{n}\right)$, is invariant under $H_{t}$. Hence, since $y$ has continuous sample paths, it is a strong Markov process and thus a diffusion (see e.g. Ito [11, p. 60]).

Let $f \in C^{2}\left(E^{n}\right)$ (= set of twice continuously differentiable functions on $E^{n}$ ). Then the infinitesimal generator for the semigroup

$$
G f(u)=\lim _{t \rightarrow 0} \frac{H_{t} f(u)-f(0)}{t}
$$

is (see e.g. Loève $[15$, p. 646])

where

$$
G f=\Sigma e_{i} \frac{\partial f}{\partial u_{i}}+\frac{1}{2} \Sigma d_{j k} \frac{\partial^{2} f}{\partial u_{j} \partial u_{k}}
$$

and

$$
\begin{aligned}
e_{i}(u) & =\lim _{t \rightarrow 0} \frac{E_{u}(y(t)-y(0))_{i}}{t} \\
& =\lim _{t \rightarrow 0} \frac{\left(e^{A t} u-u\right)_{i}}{t}=(A u)_{i} \\
& = \begin{cases}u_{i+1}, & i=1, \cdots, n-1, \\
a_{n} u_{1}+\cdots+a_{1} u_{n}, & i=n\end{cases}
\end{aligned}
$$

$$
\begin{aligned}
d_{j k}(u) & =\lim _{t \rightarrow 0} \frac{E_{u}\left[(y(t)-y(0))_{j}(y(t)-y(0))_{k}\right]}{t} \\
& =\lim _{t \rightarrow 0} \frac{E_{u}\left[\left(y(t)-e^{A t} y(0)\right)_{j}\left(y(t)-e^{A t} y(0)\right)_{k}\right]}{t} \\
& =\lim _{t \rightarrow 0} \frac{R_{j k}(t)}{t} \\
& = \begin{cases}1 & \text { if } j=k=n, \\
0 & \text { otherwise. }\end{cases}
\end{aligned}
$$

Hence the (infinitesimal) generator for the trajectory process arising from the differential operator $P(D)=D^{n}-a_{1} D^{n-1}-\cdots-a_{n}$ is

$$
G=\frac{1}{2} \frac{\partial^{2}}{\partial u_{n}^{2}}+\sum_{i=1}^{n-1} u_{i+1} \frac{\partial}{\partial u_{i}}+\left(u_{1} a_{n}+\cdots+u_{n} a_{1}\right) \frac{\partial}{\partial u_{n}} .
$$

Correspondingly the adjoint to the generator,

$$
G^{*}=\frac{1}{2} \frac{\partial^{2}}{\partial u_{n}^{2}}-\sum_{i=1}^{n-1} u_{i+1} \frac{\partial}{\partial u_{i}}-\left(u_{1} a_{n}+\cdots+u_{n} a_{1}\right) \frac{\partial}{\partial u_{n}}-a_{1} .
$$


REMARK 2.3. In the theory of stochastic differentials, Ito [11, pp. 172-210], a prescription is developed which enables one to easily write down the generator $G$ corresponding to a stochastic differential equation such as (2.1). Conversely this prescription quickly yields the stochastic differential equations corresponding to a given generator.

It is worth noting that the differential operators $G$ and $G^{*}$ are "singular" elliptic. That is to say if $e_{i k}$ denotes the coefficient of $\partial^{2} / \partial u_{i} \partial u_{k}$ then the matrix $\left(e_{t k}\right)$ is nonnegative definite and the determinant of $\left(e_{i k}\right)$ is zero. Consequently virtually all the results of the theory of elliptic equations are not directly applicable.

3. Properties of the trajectory process $y$. Up to this point we have shown that the trajectory process $y$ is a Gaussian diffusion. A list of additional properties of the $y$ process will be presented below. For future convenience the list is phrased in terms of a general diffusion process.

Let $x$ be a diffusion process defined on $[0, \infty) \times \Omega^{\prime}$ and taking values in $S$ where $\Omega^{\prime}$, the path space, is the set of all continuous $S$ valued functions on $[0, \infty)$ and $S$ is a regular locally compact Hausdorff space which satisfies the second axiom of countability. Let the statistical behavior of $x$ be governed by the family of stationary transition probability measures $P_{c}^{\prime}(t, U)$ which are defined for every $t \geqq 0$, every $c \in S$ and every Borel set $U \subset S$. Let $H_{t}^{\prime}, t \geqq 0$, denote the corresponding semigroup of transition operators defined on the class of bounded measurable real valued functions on $S, \mathscr{B}(S)$, by

$$
H_{t}^{\prime} f(a)=\int_{S} P_{a}^{\prime}(t, d b) f(b) .
$$

We shall say that the process $x=\left([0, \infty) \times \Omega^{\prime}, S,\left\{P_{a}^{\prime}: a \in S\right\}\right)$ satisfies

(P.1) if for every $t>0, H_{t}^{\prime}: \mathscr{B}(S) \rightarrow C(S)$; the set of continuous functions on $S$,

(P.2) if for every $t>0$ and every Borel set $U \subset S, P^{\prime} \cdot(t, U)$ is continuous on $S$,

(P.3) if for every $t>0$, every nonempty set $U$ in $S$ and every $c \in S, P_{c}^{\prime}(t, U)>0$,

(P.4) (extremal property) if the only $f \in \mathscr{B}(S)$ which satisfies the conditions:

(1) $m \leqq f \leqq M$;

(2) $H_{t} f=f$;

(3) there exists a point $a \in S$ such that $f(a)=M(m)$;

is the constant function $f \equiv M(m)$.

Define the hitting time to an open set $V, \tau_{V}$, as

$$
\begin{aligned}
\tau_{V}(\omega) & =\inf [t \geqq 0: x(t, \omega) \in V] & & \text { if such a } t \text { exists, } \\
& =+\infty & & \text { otherwise. }
\end{aligned}
$$

Let $U$ be any open set with closure $\bar{U}$ and nonempty exterior $V=\bar{U}^{c}$. We say $x$ satisfies 
(P.5) if for each $a \in U$ there exists a neighborhood $N$ of $a$ and a pair of positive constants $q$ and $k$ such that for every $c \in N$,

$$
P_{c}^{\prime}\left(\tau_{V}<t\right) \leqq q e^{-k / t},
$$

(P.6) if $P^{\prime}\left(\tau_{V}<\infty\right)$ is continuous on $U$,

(P.7) if $E^{\prime}\left(\tau_{V}<\infty\right)$ is continuous on $U$ whenever $\bar{U}$ is compact.

THEOREM 3.1. The trajectory process $y=\left([0, \infty) \times \Omega, E^{n}, \quad\left\{P_{a}: a \in E^{n}\right\}\right)$

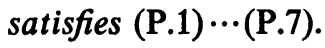

Proof. Theorem 2.1 implies that $y$ satisfies (P.1) and (P.2). For every $t>0$ and every $a \in E^{n}$ the measure $P_{a}(t, d c)$ is absolutely continuous with respect to Lebesgue measure and has a density

$$
p(t ; a, c)=(2 \pi)^{-n / 2}|R(t)|^{-1 / 2} \exp \left\{-\frac{1}{2}\left\langle c-e^{A t} a, R^{-1}(t)\left(c-e^{A t} a\right)\right\rangle\right\}
$$

which is positive everywhere. Hence $y$ satisfies (P.3); (P.4) is an easy consequence of (P.1) and (P.3). Theorem 3.2 and a Corollary serve to establish (P.5), (P.6) and (P.7).

It is expedient to first prove

LEMMA 3.1. Let $W$ be a bounded subset of $E^{n}$ and let $T$ be a positive number. Then there exist a pair of positive constants $K$ and $M$ such that for every $0<t \leqq T$

$$
p(t ; a, b) \leqq K|R(t)|^{-1 / 2} \exp \left(-M\|b-a\|^{2} / t\right)
$$

whenever either $(a, b) \in\left(W \times E^{n}\right)$ or $(a, b) \in\left(E^{n} \times W\right)$.

Proof. We shall suppose that $b \in W$, the other case may be treated in a similar fashion. Now

$$
\Sigma\left(b-e^{A t} a\right)_{i} R_{i j}^{-1}(t)\left(b-e^{A t} a\right)_{j} \geqq \eta(t)\left\|e^{-A t} b-a\right\|^{2}
$$

where $\eta(t)$ is the minimum eigenvalue of the matrix $\left(e^{A t}\right)^{*} R^{-1}(t) e^{A t}$. If we set $\phi_{i}(s)=\left(e^{A s}\right)_{i n}$ then the maximum eigenvalue of the inverse to this matrix is equal to

$$
\max _{x \neq 0} \int_{0}^{t} \frac{\left(\sum x_{i} \phi_{i}(-s)\right)^{2} d s}{\sum x_{i}^{2}} \leqq t / 4 M
$$

for some $M>0$ when $0 \leqq t \leqq T$. Hence

$$
\begin{aligned}
\eta(t)\left\|e^{-A t} b-a\right\|^{2} & \geqq(4 M / t)\left\|e^{-A t} b-a\right\|^{2} \\
& \geqq(2 M / t)\|b-a\|^{2}-(4 M / t)\left\|e^{-A t} b-b\right\|^{2} .
\end{aligned}
$$

Since the last term on the right is bounded for $b \in W$ the desired result follows easily. 
The notation $\omega_{t}^{+}$which denotes the shifted path $\omega_{t}^{+}(s)=\omega(t+s)$ will be useful in the next proof.

THEOREM 3.2. Let $U$ be an open set with closure $\bar{U}$. Suppose $a \in U$ and $V=\bar{U}^{c}$ is nonempty. Then there exists a neighborhood $N$ of $a$ and a pair of positive constants $M$ and $k$ such that for every $c \in N$

$$
P_{c}\left(\tau_{V}<t\right) \leqq M e^{-k / t} .
$$

Proof. It suffices to establish the inequality when $\bar{U}$ is compact. Choose $c \in U$ and $\delta>0$ such that the distance from $c$ to $V$ is $>2 \delta$. Lemma 3.1 implies that there exist positive constants $q, k, T$ such that

$$
P_{b}[\|y(s)-b\|>\delta] \leqq q e^{-k / s}<1 / 2
$$

for all $b \in U$ and $0 \leqq s \leqq T$. Let $\tau_{c}$ denote the first exit time out of a sphere of radius $2 \delta$ about $c$. Then for $t<T$

$$
\begin{aligned}
q e^{-k / t} & \geqq P_{c}(\|y(t)-c\|>\delta) \geqq P_{c}\left(\tau_{c}<t,\|y(t)-c\|>\delta\right) \\
& =P_{c}\left[\omega: \tau_{c}(\omega)<t,\left\|y\left(t-\tau_{c}(\omega), \omega_{\tau_{c}}^{+}\right)-c\right\|>\delta\right] \\
& =E_{c}\left[\omega: \tau_{c}(\omega)<t ; P_{y\left(\tau_{c}(\omega), \omega\right)}\left(\omega^{\prime}:\left\|y\left(t-\tau_{c}(\omega), \omega^{\prime}\right)-c\right\|>\delta\right)\right] \\
& \geqq E_{c}\left[\omega: \tau_{c}(\omega)<t ; P_{y\left(\tau_{c}(\omega), \omega\right)}\left(\omega^{\prime}:\left\|y\left(t-\tau_{c}(\omega), \omega^{\prime}\right)-y\left(0, \omega^{\prime}\right)\right\|<\delta\right)\right] \\
& \geqq(1 / 2) P_{c}\left(\tau_{c}<t\right) .
\end{aligned}
$$

This estimate is valid for all $c \in U$. But if $c \in U$ and also the distance from $c$ to $V$ exceeds $2 \delta$ then $P_{c}\left(\tau_{c}<t\right) \geqq P_{c}\left(\tau_{V}<t\right)$. The theorem follows easily.

COROLlaRY 1. Let $U$ be any open set with closure $\bar{U}$ and nonempty exterior $\bar{U}^{c}=V$. The the function $p(\cdot)=P .\left(\tau_{V}<\infty\right)$ is continuous on $U$. If $\bar{U}$ is compact then the function $g(\cdot)=E .\left(\tau_{V}\right)$ is also continuous on $U$.

Proof. Consider first $p$ and note that for any $c \in U$

$$
\begin{aligned}
& p(c) \geqq H_{t} p(c)=P_{c}\left(\tau_{V}\left(\omega_{t}^{+}\right)<\infty\right) \\
& \geqq P_{c}\left(\tau_{V}\left(\omega_{t}^{+}\right)<\infty, \tau_{V}>t\right) \\
& \\
&=p(c)-P_{c}\left(\tau_{V}<\infty, \tau_{V} \leqq t\right) \\
& \geqq p(c)-P_{c}\left(\tau_{V}<t\right) .
\end{aligned}
$$

Hence

$$
\left|p(c)-H_{t} p(c)\right| \leqq P_{c}\left(\tau_{V}<t\right)
$$

and thus by Theorem 3.2 the continuous functions $H_{t} p$ converge uniformly to $p$ locally on $U$ as $t \rightarrow 0$. Therefore $p$ is continuous on $U$. 
The proof for $g$ proceeds in a similar vein. First note that

$$
\begin{aligned}
\left|g(c)-H_{t} g(c)\right| & \leqq t+E_{c}\left[\tau_{V}\left(\omega_{t}^{+}\right) ; \tau_{V}<t\right] \\
& =t+E_{c}\left[E_{y(t)}\left(\tau_{V}\right) ; \tau_{V}<t\right] \\
& \leqq t+M P_{c}\left(\tau_{V}<t\right)
\end{aligned}
$$

where, since $\bar{U}$ is compact, $M=\sup _{a \in \bar{U}} E_{a}\left(\tau_{V}\right)<\infty$ (Dynkin [9, Lemma 4, p. 41]). Hence the continuous functions $H_{t} g$ converge uniformly to $g$ locally on $U$ as $t \rightarrow 0$ and so $g$ must be continuous on $U$.

4. Characterization of transience and recurrence in terms of average sojourn time. In this section we shall find necessary and sufficient conditions for a general diffusion process $x=\left([0, \infty) \times \Omega^{\prime}, S,\left\{P_{a}^{\prime}: a \in S\right\}\right)$ to be either transient or recurrent in terms of the average amount of time the process spends in "bounded" open sets; that is open sets with compact closure. We start with the following definitions:

Definition 4.1. A diffusion process $x$ is recurrent if and only if $P_{c}^{\prime}\left(\tau_{\boldsymbol{V}}<\infty\right)=1$ for every $c \in S$ and every nonvoid open set $V \subset S$.

Definition 4.2. A diffusion process $x$ is transient if and only if $\lim _{n \rightarrow \infty} P_{c}^{\prime}\left(\tau_{V}\left(\omega_{n}^{+}\right)<\infty\right)=0$ for every $c \in S$ and every open set $V \subset S$ with compact closure.

Theorem 4.1. If the diffusion process $x$ satisfies (P.2), (P.3), (P.6) and (P.7) then it is recurrent if and only if the average sojourn time in every nonempty open set is infinite.

Proof. Suppose first that the process is recurrent. Let $U$ be a nonempty open set and choose $U_{0} \subset \bar{U}_{0} \subset U_{1} \subset \bar{U}_{1} \subset U$ such that $U_{0}$ and $U_{1}$ are open nonempty sets and $\bar{U}_{1}$ is compact. Let $V=\bar{U}_{1}^{c}$. Define the sequence of hitting times

$$
\begin{aligned}
\tau_{1} & =\tau_{U_{0}}, \\
\tau_{2 n} & = \begin{cases}\tau_{2 n-1}+\tau_{V}\left(\omega_{\tau_{2 n-1}}^{+}\right) & \text {if } \tau_{2 n-1}<\infty, \\
+\infty & \text { otherwise, }\end{cases} \\
\tau_{2 n+1} & = \begin{cases}\left.\tau_{2 n}+\tau_{U_{0}}\left(\omega_{\tau_{2 n}}^{+}\right)\right) & \text {if } \tau_{2 n}<\infty, \\
+\infty & \text { otherwise } n=1,2, \cdots\end{cases}
\end{aligned}
$$

and let $\sigma_{U}(\omega)$ denote the sojourn time in the set $U$ :

$$
\sigma_{U}(\omega)=\int_{0}^{\infty} \chi_{U}(x(t, \omega)) d t,
$$

where $\chi_{U}$ denotes the characteristic function of the set $U$. Then 


$$
\begin{aligned}
E_{a}^{\prime}\left(\sigma_{U}\right) & \geqq \sum_{n=1}^{\infty} E_{a}^{\prime}\left[\tau_{2 n}-\tau_{2 n-1} ; \tau_{2 n-1}<\infty\right] \\
& =\sum_{n=1}^{\infty} E_{a}^{\prime}\left[\tau_{V}\left(\omega_{\tau_{2 n-1}}^{+}\right) ; \tau_{2 n-1}<\infty\right] \\
& =\sum_{n=1}^{\infty} E_{a}^{\prime}\left[E_{x \tau_{2 n-1}}^{\prime}\left(\tau_{V}\right) ; \tau_{2 n-1}<\infty\right] .
\end{aligned}
$$

Since $E^{\prime} \cdot\left(\tau_{V}\right)$ is a positive continuous function on the compact boundary of $U_{0}$ there exists a number $\gamma$ such that for every $b$ belonging to the boundary of $U_{0}$

Hence

$$
E_{b}^{\prime}\left(\tau_{V}\right) \geqq \gamma>0 \text {. }
$$

$$
E_{a}^{\prime}\left(\sigma_{U}\right) \geqq \gamma \sum_{n-1}^{\infty} P_{a}^{\prime}\left(\tau_{2 n-1}<\infty\right)=+\infty .
$$

The divergence of the sum is a consequence of the assumed recurrence which implies $P_{a}^{\prime}\left(\tau_{2 n-1}<\infty\right)=1$, as may easily be verified by induction.

Suppose now that the process is not recurrent. Then there exists a nonempty open set $W$ with compact closure and a point $a \in W^{c}$ such that $P_{a}^{\prime}\left(\tau_{W}<\infty\right)<1$. We show, following Khasminskii [14], that $E_{b}^{\prime}\left(\sigma_{W}\right)<\infty$ for every $b \in S$. Since $P^{\prime}\left(\tau_{W}<\infty\right)$ is continuous on $W^{c}$ we can choose a compact neighborhood $N$ of $a$ such that $q=\sup _{b \in N} P_{b}^{\prime}\left(\tau_{W}<\infty\right)<1$. But, for $T>0$

hence

$$
\left[\omega: \sigma_{W}(\omega)>T\right] \subset\left[\omega: \tau_{W}\left(\omega_{T}^{+}\right)<\infty\right],
$$

$$
\begin{aligned}
P_{c}^{\prime}\left(\sigma_{W}>T\right) & \leqq P_{c}^{\prime}\left(\tau_{W}\left(\omega_{T}^{+}\right)<\infty\right) \\
& =\int_{S} P_{c}^{\prime}(T, d e) P_{e}^{\prime}\left(\tau_{W}<\infty\right) \\
& \leqq q P_{c}^{\prime}(T, N)+1-P_{c}^{\prime}(T, N) \\
& =1-(1-q) P_{c}^{\prime}(T, N) .
\end{aligned}
$$

However, (P.2) and (P.3) imply that $P^{\prime}(T, N)$ is a continuous strictly positive function. Therefore there exists a number $\beta>0$ such that

$$
P_{c}^{\prime}\left(\sigma_{W}>T\right) \leqq \beta<1
$$

for every $c \in W$.

For any point $b$

where

$$
P_{b}^{\prime}\left(\sigma_{W}>2 T\right)=P_{b}^{\prime}\left(\sigma_{W}>T, \sigma_{W}\left(\omega_{m(T)}^{+}\right)>T\right),
$$

$$
m(t)=\left\{\begin{array}{lc}
\inf [r>0: t= & \left.\int_{0}^{r} \chi_{W}(x(s)) d s\right] \text { if such an } r \text { exists } \\
+\infty & \text { otherwise }
\end{array}\right.
$$


Note that $m(t)$ is a Markov time as follows from the equality:

Since $x(m(T)) \in W$

$$
[m(t)>T]=\left[\int_{0}^{T} \chi_{W}(x(s)) d s<t\right] .
$$

Iterating we find

$$
\begin{aligned}
P_{b}^{\prime}\left(\sigma_{W}>2 T\right) & =E_{b}^{\prime}\left[P_{x(m(T))}^{\prime}\left(\sigma_{W}>T\right) ; \sigma_{W}>T\right] \\
& \leqq \beta P_{b}^{\prime}\left(\sigma_{W}>T\right) \leqq \beta .
\end{aligned}
$$

and thus

$$
P_{b}^{\prime}\left(\sigma_{W}>n T\right) \leqq \beta^{n-1}
$$

$$
E_{b}^{\prime}\left(\sigma_{W}\right)=\int_{0}^{\infty} P_{b}^{\prime}\left(\sigma_{W}>t\right) d t<\infty .
$$

This completes the proof.

Now, for any point $a \in S$ and any open set, with compact closure, $B \subset S$ define

$$
h_{B}(a)=\lim _{n \rightarrow \infty} P_{a}^{\prime}\left(\tau_{B}\left(\omega_{n}^{+}\right)<\infty\right) .
$$

We establish a zero one law.

THEOREM 4.2. If $x$ is a diffusion process which satisfies (P.1) and (P.4) then either $h_{B} \equiv 1$ or $h_{B} \equiv 0$.

Proof. Clearly $0 \leqq h_{B} \leqq 1$. A direct application of the Lebesgue bounded convergence theorem leads to the equality

$$
\begin{aligned}
H_{t}^{\prime} h_{B}(a) & =\lim _{n \rightarrow \infty} E_{a}^{\prime}\left(P_{x(t)}^{\prime}\left(\tau_{B}\left(\omega_{n}^{+}\right)<\infty\right)\right) \\
& =\lim _{n \rightarrow \infty} P_{a}^{\prime}\left(\tau_{B}\left(\omega_{n+t}^{+}\right)<\infty\right) \\
& =h_{B}(a) .
\end{aligned}
$$

Hence, by (P.1), $h_{B}$ is continuous and, by (P.4), if there exists a point $b \in S$ for which $h_{B}(b)=1$ then $h_{B} \equiv 1$. Conversely suppose that $h_{B}<1$. Since $h_{B}$ is continuous and $\bar{B}$ is compact there exists a positive number $\theta$ such that for every $b \in \bar{B}$,

Hence

$$
h_{B}(b) \leqq \theta<1
$$

$$
\begin{aligned}
h_{B}(a) & =\lim _{n} P_{a}^{\prime}\left(\tau_{B}\left(\omega_{n}^{+}\right)<\infty\right) \\
& =\lim _{n} P_{a}^{\prime}\left(\tau_{B}\left(\omega_{n+k}^{+}\right)<\infty\right) \\
& =\lim _{n} P_{a}^{\prime}\left(\tau_{B}\left(\omega_{n+k}^{+}\right)<\infty, \tau_{B}\left(\omega_{k}^{+}\right)<\infty\right) \\
& =\lim _{n} E_{a}^{\prime}\left[P_{x\left(\tau_{B}\left(\omega_{k}^{+}\right)+k\right)}^{\prime}\left(\tau_{B}\left(\omega_{n}^{+}\right)<\infty\right) ; \tau_{B}\left(\omega_{k}^{+}\right)<\infty\right] \\
& =E_{a}^{\prime}\left[h\left(x\left(\tau_{B}\left(\omega_{k}^{+}\right)+k\right) ; \tau_{B}\left(\omega_{k}^{+}\right)<\infty\right]\right. \\
& \leqq \theta P_{a}^{\prime}\left(\tau_{B}\left(\omega_{k}^{+}\right)<\infty\right) .
\end{aligned}
$$


Taking the limit as $k \rightarrow \infty$, this implies that

$$
h_{B}(a) \leqq \theta h_{B}(a) \text {. }
$$

But this is only possible if $h_{B} \equiv 0$.

Since $P_{a}^{\prime}\left(\tau_{B}<\infty\right) \geqq h_{B}(a)$ the following result is now easily established.

THEOREM 4.3. Suppose the diffusion process $x$ satisfies (P.1) and (P.4). If

(1) $h_{B}(a)=1$ for some $a \in E^{n}$ then $P^{\prime}\left(\tau_{B}<\infty\right) \equiv 1$,

(2) $P^{\prime}\left(\tau_{B}<\infty\right) \equiv 1$ then $h_{B} \equiv 1$.

THEOREM 4.4. Suppose the diffusion process $x$ satisfies (P.1), (P.2), (P.3), (P.4), (P.6) and (P.7). Then the process is transient if and only if the average sojourn time in every compact set is finite.

Proof. It suffices to consider open sets with compact closure. Suppose first the process is transient and let $U$ be any open set with compact closure. Theorem 4.3 implies that there exists at least one point $a$ for which $P_{a}^{\prime}\left(\tau_{U}<\infty\right)<1$. Hence by (the second half of the proof of) Theorem 4.1 the average sojourn time in $U$ is finite.

Conversely, suppose the average sojourn time in every open set with compact closure is finite. Then if $V$ is any such set there exists by (the first half of the proof of) Theorem 4.1 a point $a$ for which $P_{a}^{\prime}\left(\tau_{V}<\infty\right)<1$. Consequently, by Theorems 4.2 and $4.3, h_{V} \equiv 0$.

5. The matrices $R(t)$ and $e^{A t}$. In this section we summarize properties of the covariance matrix, $R(t)$, and the solution matrix, $e^{A t}$, for later use. For more detail the reader is referred to Chapter 3 of Coddington and Levinson [5].

Let $\zeta_{1}(t), \cdots, \zeta_{n}(t)$ denote the fundamental solutions of the homogeneous differential equation

$$
\left(D^{n}-a_{1} D^{n-1} \cdots-a_{n}\right) y=0 .
$$

Let $Z(t)$ denote the matrix

$$
Z(t)=\left[\begin{array}{cccc}
\zeta_{1}(t) & \zeta_{2}(t) & \cdots & \zeta_{n}(t) \\
\zeta_{1}^{(1)}(t) & \zeta_{2}^{(1)}(t) & \cdots & \zeta_{n}^{(1)}(t) \\
\vdots & \vdots & & \vdots \\
\zeta_{1}^{(n-1)}(t) & \zeta_{2}^{(n-1)}(t) & \cdots & \zeta_{n}^{(n-1)}(t)
\end{array}\right]
$$

where $\zeta^{(k)}(t)=d^{k} \zeta / d t^{k}$.

Then if $P=Z(0), P^{-1}$ exists and $P^{-1} A P=J$ where $J$ is a matrix in Jordan form. Furthermore

and consequently

$$
e^{A t}=Z(t) Z^{-1}(0)=Z(t) P^{-1}
$$

$$
e^{J t}=P^{-1} e^{\Lambda t} P=P^{-1} Z(t) .
$$


The Green function discussed in Remark 2.1

$$
g(s)=\left(e^{A s}\right)_{1 n}=\sum_{i=1}^{n} P_{i n}^{-1} \zeta_{i}(s) \quad(s \geqq 0)
$$

where all terms $P_{i n}^{-1}, i=1, \cdots, n$, are nonzero.

The functions

$$
\phi_{i}(s)=\left(e^{A s}\right)_{\text {in }}=g^{(i-1)}(s) \text { for } s \geqq 0,
$$

$i=1, \cdots, n$, are linearly independent on every interval as are also the functions

$$
\psi_{i}(s)=\left(e^{J s} P^{-1}\right)_{i n} \quad i=1, \cdots, n .
$$

Recall that

$$
R_{i j}(t)=\int_{0}^{t} \phi_{i}(s) \phi_{j}(s) d t .
$$

The determinant, $|R(t)|$, is a monotone (strictly) increasing positive real valued analytic function of $t$ for $0<t<\infty$. Monotonicity is an easy consequence of the extremal characterization (Bekenbach and Bellman, [1, Theorem 24, p. 76])

$$
|R(t)|=\min _{Y}\left\langle y^{(1)}, R(t) y^{(1)}\right\rangle \cdots\left\langle y^{(n)}, R(t) y^{(n)}\right\rangle
$$

where the minimization is performed over all orthogonal matrices $Y$, whose columns are the orthonormal vectors $y^{(1)}, \cdots, y^{(n)}$. If $h>0$

$$
\begin{aligned}
|R(t+h)| & =\min _{Y} \prod_{i=1}^{n}\left[\int_{0}^{t}\left(\sum_{j=1}^{n} y_{j}^{(i)} \phi_{j}(s)\right)^{2}+\int_{t}^{t+h}\left(\sum_{j=1}^{(n)} y_{j}^{(i)} \phi_{j}(s)\right)^{2} d s\right] \\
& >|R(t)|
\end{aligned}
$$

since the $\phi_{j}(s), j=1, \cdots, n$ are linearly independent.

Let $P^{*}$ denote the transpose of $P$. Then

where

$$
R(t)=P U(t) P^{*}
$$

$$
U_{i j}(t)=\int_{0}^{t} \psi_{i}(s) \psi_{j}(s) d s .
$$

Clearly $U(t)$ is a symmetric positive definite matrix for $t>0$, and $|U(t)|$ is a monotone strictly increasing positive real valued analytic function of $t$ for $0<t<\infty$.

6. Conditions for the trajectory process $y$ to be recurrent or transient. We first relate the average sojourn time in a set to $\operatorname{sp}(A)$, the spectrum of the matrix $A$, for the trajectory process $y$.

Definition 6.1. We shall say that $\operatorname{sp}(A)$ is type I whenever the roots of the 
polynomial $z^{n}-a_{1} z^{n-1}-\cdots-a_{n}$ lie in $\bar{\Lambda}[\operatorname{Re}(z) \leqq 0]$ with at most two roots on the boundary $[\operatorname{Re}(z)=0]$ and no multiple roots on the boundary.

THEOREM 6.1. If $\operatorname{sp}(A)$ is type I then the average sojourn time of the process $y$ in every open nonempty set is infinite. If $\operatorname{sp}(A)$ is not type I then the average sojourn time of the process $y$ in every compact set is finite.

Recall that the average sojourn time inside a set $B$ is equal to

$$
E_{a}\left(\sigma_{B}\right)=E_{a} \int_{0}^{\infty} \chi_{B}(y(t, \omega)) d t=\int_{0}^{\infty} P_{a}(t, B) d t .
$$

This theorem is then an easy consequence of the following two lemmas.

Lemma 6.1. Suppose that $\operatorname{sp}(A)$ is type I. Then for any $a, b \in\left(E^{n}\right)$

$$
\int_{1}^{\infty} p(t, a, b) d t=+\infty
$$

Proof. Let $c(t)=b-e^{A t} a$. Then

But

$$
(2 \pi)^{n / 2} p(t, a, b)=|R(t)|^{-1 / 2} \exp \left[-\frac{1}{2}\left\langle c(t), R^{-1}(t) c(t)\right\rangle\right]
$$

where

$$
\left\langle c(t), R^{-1}(t) c(t)\right\rangle \leqq \lambda(t)\langle c(t), c(t)\rangle
$$

$$
\begin{aligned}
\frac{1}{\lambda(t)}=\left(\max _{y \neq 0} \frac{\left\langle y, R^{-1}(t) y\right\rangle}{\langle y, y\rangle}\right)^{-1} & =\min _{y \neq 0} \frac{\langle y, R(t) y\rangle}{\langle y, y\rangle} \\
& =\min _{y \neq 0} \frac{\int_{0}^{t}\left|\sum y_{i} \phi_{i}(s)\right|^{2} d s}{\sum y_{i}^{2}} .
\end{aligned}
$$

Since the $\phi_{i}(s)$ are linearly independent $1 / \lambda(1) \geqq 0$ and $\lambda(t) \leqq \lambda(1)$ for $t \geqq 1$. Furthermore, when $\operatorname{sp}(A)$ is type $\mathrm{I}\langle c(t), c(t)\rangle$ is bounded above. Thus there exists a positive constant $M$ such that

$$
p(t, a, b) \geqq M|R(t)|^{-1 / 2}, \quad t \geqq 1,
$$

and it suffices to prove that $\int_{1}^{\infty}|R(t)|^{-1 / 2} d t=+\infty$. Since $R(t)=P U(t) P^{*}$ it is in fact enough to show that the monotone increasing function $|U(t)|=O\left(t^{2}\right)$ as $t \rightarrow \infty$. The inequality (valid for symmetric positive-definite matrices [1, Theorem 7, p. 63])

$$
\begin{aligned}
|U(t)| \leqq U_{11}(t) \cdots U_{n n}(t) & =\prod_{i=1}^{n} \int_{0}^{t} \psi_{i}^{2}(s) d s \\
& =\prod_{i=1}^{n} \int_{0}^{t}\left(e^{J s} P^{-1}\right)_{i n}^{2} d s
\end{aligned}
$$

may be applied to conclude that if $\operatorname{sp}(A)$ is type $\mathrm{I}$ and $\operatorname{sp}(A)$ 
(a) does not contain any boundary points then

$$
|U(t)|=O(1)(t \rightarrow \infty),
$$

(b) contains the single boundary point zero then

$$
|U(t)|=O(t)(t \rightarrow \infty),
$$

(c) contains the pair of boundary points $\pm i \beta(\beta>0)$ then

$$
|U(t)|=O\left(t^{2}\right)(t \rightarrow \infty) \text {. }
$$

LeMma 6.2. If $\operatorname{sp}(A)$ is not type I then for any $a, b \in E^{n}$

$$
\int_{1}^{\infty} p(t, a, b) d t<\infty \text {. }
$$

Proof. It clearly suffices to show that $\int_{1}^{\infty}|U(t)|^{-1 / 2} d t<\infty$. There are three cases to consider:

(a) two eigenvalues of $A$ are identically zero, all the remaining eigenvalues have negative real parts,

(b) three or more eigenvalues have zero real part, the remainder have negative real parts,

(c) one or more eigenvalues of $A$ have positive real parts.

Consider first case (a).

$$
|U(t)|=\prod_{i=1}^{n} \lambda_{i}(t)
$$

where $\lambda_{i}(t)$ are the eigenvalues of $U(t)$ ordered so that

Clearly

$$
\lambda_{1}(t) \geqq \lambda_{2}(t) \geqq \cdots \geqq \lambda_{n}(t) .
$$

$$
|U(t)| \geqq \lambda_{1}(t)\left(\lambda_{n}(t)\right)^{n-1}
$$

But

$$
\begin{aligned}
\lambda_{1}(t) & =\max _{y \neq 0} \frac{\langle y, U(t) y\rangle}{\langle y, y\rangle}=\max _{y \neq 0} \frac{\int_{0}^{t}\left|\Sigma y_{i} \psi_{i}(s)\right|^{2} d s}{\Sigma y_{i}^{2}} \\
& \geqq \max _{i} \int_{0}^{t}\left|\psi_{i}(s)\right|^{2} d s .
\end{aligned}
$$

There exists however, for case (a), a choice of $i$ for which

$$
\psi_{i}(s)=b_{1}+b_{2} s \quad b_{1} \neq 0, b_{2} \neq 0,
$$

and hence there exists a positive constant $k_{1}$ such that

$$
\lambda_{1}(t) \geqq k_{1} t^{3} \text { for } t \geqq 1 \text {. }
$$


Since also

$$
\lambda_{n}(t)=\min _{y \neq 0} \frac{\langle y, U(t) y\rangle}{\langle y, y\rangle}
$$

is monotone increasing there exists a positive constant $k_{2}$ such that

$$
|U(t)| \geqq k_{2} t^{3} \quad \text { for } t \geqq 1
$$

and hence $\int_{1}^{\infty}|U(t)|^{-1 / 2} d t<\infty$.

A similar argument applies to case (c); in fact in this case $\lambda_{1}(t)$ approaches $\infty$ exponentially fast. It remains to consider case (b).

Suppose first that exactly three eigenvalues of $A$ have zero real part. Since that characteristic equation has real coefficients we may assume these three eigenvalues to be distinct; for, if two agree all three must agree and equal zero, in which case convergence follows from case (a). Let us denote these eigenvalues by $+i \beta$, $-i \beta$ and $0(\beta>0)$ and assume that the Jordan form is so ordered that

Then

$$
e^{J s}=\left[\begin{array}{ccccc}
\cos \beta s & 0 & 0 & \cdots & 0 \\
0 & \sin \beta s & 0 & \cdots & 0 \\
0 & 0 & 1 & \cdots & 0 \\
0 & 0 & 0 & & \\
\vdots & \vdots & \vdots & & \\
0 & 0 & 0 & &
\end{array}\right] .
$$

$$
\begin{aligned}
& \psi_{1}(s)=\left(e^{J s} P^{-1}\right)_{1 n}=P_{1 n}^{-1} \cos \beta s \\
& \psi_{2}(s)=\left(e^{J s} P^{-1}\right)_{2 n}=P_{2 n}^{-1} \sin \beta s \\
& \psi_{3}(s)=\left(e^{J s} P^{-1}\right)_{3 n}=P_{3 n}^{-1} .
\end{aligned}
$$

(Recall that all the entries in the $n$th column of $P^{-1}$ are nonzero.)

Now since

$$
|U(t)|=\lambda_{1}(t) \lambda_{2}(t) \cdots \lambda_{n}(t) \geqq \lambda_{1}(t) \lambda_{2}(t) \lambda_{3}(t)\left[\lambda_{n}(t)\right]^{n-3}
$$

and $[1$, Theorem 23, p. 76$]$

$$
\lambda_{1}(t) \lambda_{2}(t) \lambda_{3}(t) \geqq\left[\begin{array}{lll}
\int_{0}^{t}\left(\psi_{1}(s)\right)^{2} d s & \int \psi_{1} \psi_{2} & \int \psi_{1} \psi_{3} \\
\int \psi_{2} \psi_{1} & \int \psi_{2} \psi_{2} & \int \psi_{2} \psi_{3} \\
\int \psi_{3} \psi_{1} & \int \psi_{3} \psi_{2} & \int \psi_{3} \psi_{3}
\end{array}\right],
$$

an easy estimate shows that there exists a $k_{3}>0$ such that

$$
\lambda_{1}(t) \lambda_{2}(t) \lambda_{3}(t) \geqq k_{3} t^{3} \text { for } t \geqq 1 \text {. }
$$


Consequently

$$
|U(t)| \geqq k_{3} t^{3}\left[\lambda_{n}(1)\right]^{n-3}
$$

and so the integral converges.

Other instances of case (b) may be treated similarly. We have the following

Corollary 1. $\int_{1}^{\infty}|R(t)|^{-1 / 2} d t<\infty$ if and only if $\operatorname{sp}(A)$ is not type $\mathrm{I}$.

We summarize the implications of Theorem 4.1, 4.4 and 6.1 in

THEOREM 6.2. The process $y$ is either recurrent or transient. The following are equivalent

(1) $y$ is recurrent,

(2) $\operatorname{sp}(A)$ is type $\mathrm{I}$,

(3) $\int_{1}^{\infty}|R(t)|^{-1 / 2} d t=+\infty$.

7. Existence and uniqueness of invariant densities. A nonnegative Borel measurable function $f$, defined on $E^{n}$, is said to be an invariant density for the process $y$ if for every $t>0$ and every $a \in E^{n}$

$$
\int_{E^{n}} f(a) p(t ; a, b) d a=f(b)
$$

or equivalently if for every $t>0, H_{t}^{*} f=f$.

We first establish the existence of invariant densities.

THEOREM 7.1. If $\operatorname{sp}(A) \subset \bar{\Lambda}(=C L H P)$ then

$$
\begin{aligned}
\psi(b) & =\lim _{t \rightarrow \infty}|R(t)|^{1 / 2}(2 \pi)^{n / 2} p(t ; 0, b) \\
& =\exp \left(-\Sigma Q_{i j} b_{i} b_{j}\right)
\end{aligned}
$$

is an invariant density for the process $y$.

If $\operatorname{sp}(A) \subset \Lambda$ then

$$
Q_{i j}=\lim _{t \rightarrow \infty} R_{i j}^{-1}(t) / 2=0 \text { if } i+j \equiv 1(\bmod 2)
$$

and the invariant density $\psi$ is unique up to a multiplicative constant.

Proof. We first prove a series of lemmas.

LEMMA 7.1. $R_{i j}^{-1}(t)$ approaches a finite limit as $t \rightarrow \infty$.

Proof. $R^{-1}(t)$ and $\dot{R}(t)\left(\dot{R}_{i j}(t)=\phi_{i}(t) \phi_{j}(t)\right)$ are both symmetric positive definite matrices. Hence

$$
\begin{aligned}
\frac{d}{d t}\left\langle c, R^{-1}(t) c\right\rangle & =-\left\langle c, R^{-1}(t) \dot{R}(t) R^{-1}(t) c\right\rangle \\
& \leqq 0
\end{aligned}
$$


Thus if $c \neq 0,\left\langle c, R^{-1}(t) c\right\rangle$ is a positive monotone decreasing function of time and must therefore approach a finite nonnegative limit as $t \rightarrow \infty$. Since $R^{-1}(t)$ is symmetric

$$
\left\langle c+b, R^{-1}(t)(c+b)\right\rangle=\left\langle c, R^{-1}(t) c\right\rangle+2\left\langle c, R^{-1}(t) b\right\rangle+\left\langle b, R^{-1}(t) b\right\rangle .
$$

Therefore $\left\langle c, R^{-1}(t) b\right\rangle$ must also converge to a finite limit as $t \rightarrow \infty$. The existence of $\lim _{t \rightarrow \infty} R_{i j}^{-1}(t)$ then follows by suitable choice of $c$ and $b$.

REMARK 7.1. If $\operatorname{sp}(A) \subset \Lambda$ then $R_{i j}(t)$ will converge to a finite limit as $t \rightarrow \infty$. If this restriction on the spectrum is removed $R_{i j}(t)$ may not converge to any limit as $t \rightarrow \infty$. For example if $n=2, a_{1}=0$ and $a_{2}=-\beta^{2} \neq 0$ then $R_{12}(t)$ $=(1-\cos 2 \beta t) / 4 \beta^{2}$ which fails to converge to any limit as $t \rightarrow \infty$ while $R_{22}(t)$ $=(2 \beta t+\sin 2 \beta t) / 4 \beta$ diverges to $+\infty$.

LEMMA 7.2. If $\operatorname{sp}(A) \subset \bar{\Lambda}$ then for any fixed real number $t$

$$
\lim _{s \rightarrow \infty} \frac{|R(t+s)|}{|R(s)|}=1 \text {. }
$$

Proof. If $\operatorname{sp}(A) \subset \bar{\Lambda}$ then for large $t$

$$
|R(t)|=p(t)+\Sigma p_{k}(t) \sin \beta_{k} t+\Sigma g_{k}(t) \cos \beta_{k} t+o\left(t^{-N}\right)
$$

where $p(t), p_{k}(t)$ and $g_{k}(t)$ are polynomials, the $\left\{\beta_{k}\right\}$ is a finite collection of nonzero real numbers and $N$ may be chosen arbitrarily large. If $p_{k}$ and $g_{k}$ are not both identically zero then

$$
p(t) /\left(p_{k}(t)+g_{k}(t)\right)=O\left(t^{m}\right)
$$

for some $m \geqq 1$ because $|R(\cdot)|$ is monotone. Hence $|R(t)| \sim c t^{0}$ as $t \rightarrow \infty$ for some nonnegative integer $v$, namely the degree of $p$, and the lemma follows.

LEMMA 7.3. If $\operatorname{sp}(A) \subset \Lambda$ then

$$
Q_{i j}=\lim _{i \rightarrow \infty} R_{i j}^{-1}(t) / 2=0 \text { if } i+j \equiv 1(\bmod 2) .
$$

Proof. Applying formula (2.4) it is easily seen, for example, that

$$
\begin{aligned}
R_{i, t+3}(t) & =\int_{0}^{t} g^{(i-1)} g^{(i+2)}=\int_{0}^{t} \frac{d}{d s}\left[g^{(i-1)} g^{(i+1)}-\left(\frac{1}{2}\right) g^{(i)} g^{(i)}\right] \\
& =g^{t-1}(t) g^{i+1}(t)-\left(\frac{1}{2}\right) g^{(i)}(t) g^{(i)}(t) \rightarrow 0
\end{aligned}
$$

as $t \rightarrow \infty$. Similar arguments imply that $R_{i j}(t) \rightarrow 0$ as $t \rightarrow \infty$ whenever $i+j \equiv 1$ $(\bmod 2)$. Hence $R_{i i}^{-1}(t) \rightarrow 0$ as $t \rightarrow \infty$ whenever $i+j \equiv 1$. (Examine a typical item in the expansion of the $i, j$ cofactor and observe that if $\sigma$ denotes the "permutation" which maps 


$$
(1, \cdots, i-1, i+1, \cdots, n) \rightarrow(1, \cdots, j-1, j+1, \cdots, n)
$$

then $1+\sigma(1)+2+\sigma(2)+\cdots+n+\sigma(n) \equiv i+j(\bmod 2)$.)

To complete the proof of the theorem write

$$
\begin{aligned}
\int \psi(b) p(t ; b, c) d b & =\int \lim _{s \rightarrow \infty}|R(s)|^{1 / 2}(2 \pi)^{n / 2} p(s ; 0, b) p(t ; b, c) d b \\
& =\lim _{s \rightarrow \infty}|R(s)|^{1 / 2}(2 \pi)^{n / 2} \int p(s ; 0, b) p(t ; b, c) d b \\
& =\lim _{s \rightarrow \infty}|R(s)|^{1 / 2}(2 \pi)^{n / 2} p(s+t ; 0, c) \\
& =\psi(c) .
\end{aligned}
$$

The interchange of order of passage to the limit with integration is justified by the Lebesgue dominated convergence theorem. For

$$
|R(s)|^{1 / 2}(2 \pi)^{n / 2} p(s ; 0, b) p(t ; b, c) \leqq p(t ; b, c) \in L^{\prime}(d b)
$$

In fact

$$
\int p(t ; b, c) d b=\left|e^{A t}\right|^{-1}=\exp (-(\operatorname{trace} A) t)=e^{-a_{1} t}
$$

If $\operatorname{sp}(A) \subset \Lambda$, then in fact

$$
\psi(b)=\lim _{t \rightarrow \infty}|R(t)|^{-1 / 2}(2 \pi)^{-n / 2} p(t ; a, b)
$$

independently of $a$. Consequently if $g$ is an invariant density for the $y$ process and $\operatorname{sp}(A) \subset \Lambda$ then $g$ is integrable. For by Fatou's lemma

$$
\begin{aligned}
(2 \pi)^{-n / 2}|R(\infty)|^{-1 / 2} \psi(b) \int g(a) d a & =\int \liminf _{t \rightarrow \infty} g(a) p(t ; a, b) d a \\
& \leqq \liminf _{t \rightarrow \infty} \int g(a) p(t ; a, b) d a \\
& =g(b) .
\end{aligned}
$$

But if $g$ is integrable we can apply the dominated convergence theorem to conclude that

$$
\psi(b)\left\{(2 \pi)^{-n / 2}|R(\infty)|^{-1 / 2} \int g(a) d a\right\}=g(b) .
$$

Thus, at least in this special case, the invariant density is unique up to a multiplicative constant.

REMARK 7.2. The uniqueness proof furnished above establishes a special case of the general principle that a "decent" recurrent process should have a unique 
invariant measure. See for example, Khasminskii [14], and Maruyama and Tanaka [17]. Since we have not verified all the hypotheses invoked by Khasminskii and Maruyama and Tanaka we shall present a separate proof in $\$ 9$. We shall show, Theorem 9.2, that if the trajectory process is recurrent, i.e. if $\operatorname{sp}(A)$ is type $\mathrm{I}$, then the invariant density is unique up to a multiplicative constant.

Formula (7.1) yields information on the nature of invariant densities, namely

LEMMA 7.4. The constant function is an invariant density if and only if $a_{1}=0$. If $a_{1}>0$ the only bounded invariant density is the zero function.

Proof. The first assertion is immediate. Suppose $f$ is a bounded invariant density and $a_{1}>0$ then, by (7.1)

$$
f(b)=\int f(c) p(t ; c, b) d c \leqq\|f\|_{\infty} e^{-a_{1} t} .
$$

The second assertion then follows by letting $t \rightarrow \infty$.

We now establish a list of properties which a function must satisfy in order to be an invariant density.

THEOREM 7.2. Let $f$ be an invariant density for the process $y$. Then

(1) $f=\exp \left(O\left(\|a\|^{2}\right)\right)$ as $\|a\| \rightarrow \infty$.

(2) $f$ is real analytic.

(3) The (mixed) derivatives of $f$, of arbitrary order, grow no faster than $\exp O\left(\|a\|^{2}\right)$ as $\|a\| \rightarrow \infty$.

(4) $G^{*} f=0$.

Proof. Let $C(t)=|R(t)|^{-1 / 2}(2 \pi)^{-n / 2}$ and let $2 \lambda$ denote the maximum eigenvalue of the positive definite matrix $\left(\exp \left[A t_{1}\right]\right) * R^{-1}\left(t_{1}\right) \exp \left[A t_{1}\right], t_{1}>0$. Then

$$
\begin{aligned}
f(0) & =\int p\left(t_{1} ; a, 0\right) f(a) d a \\
& \geqq C\left(t_{1}\right) \int \exp \left(-\lambda\|a\|^{2}\right) f(a) d a .
\end{aligned}
$$

or all $t>0$, however,

$$
\begin{aligned}
f(b) & =\int p(t ; a, b) f(a) d a \\
& \leqq \exp \left(\left(\frac{1}{2}\right)\left\langle b, R^{-1}(t) b\right\rangle\right) C(t) \int \exp \left(-\left(\frac{1}{4}\right)\left\langle e^{A t} a, R^{-1}(t) e^{A t} a\right\rangle\right) f(a) d a .
\end{aligned}
$$

We can now apply Lemma 3.1 to conclude the existence of a positive constant $m$ such that for all $0<t \leqq T$

$$
f(b) \leqq \exp \left(\frac{1}{2}\left\langle b, R^{-1}(t) b\right\rangle\right) C(t) \int \exp \left(-m\|a\|^{2} / t\right) f(a) d a .
$$


Hence if $t$ is chosen so that $\lambda<m / t$ then

$$
f(b) \leqq \exp \left(\left(\frac{1}{2}\right)\left\langle b, R^{-1}(t) b\right\rangle\right) C(t) f(0) / C\left(t_{1}\right) .
$$

This establishes conclusion (1). Conclusion (3) may be established similarly.

Now let $t>0$ be fixed. Reasoning as in the proof of Lemma 2.1, we conclude that

$$
f_{N}(\cdot)=\int_{\|a\| \leqq N} p(t ; a, \cdot) f(a) d a
$$

is a real analytic function on $E^{n}$. Furthermore since

$$
\int_{E^{n}}|p(t ; a, u+i v)| f(a) d a=f(u) \exp \left(\left\langle v, R^{-1}(t) v\right\rangle / 2\right)
$$

the sequence of functions $f_{N}, N=1,2, \cdots$ is uniformly bounded and analytic on a complex neighborhood of $u$. Consequently the pointwise limit $f$ must be analytic.

Let $\phi$ be any positive $C^{\infty}$ function on $E^{n}$ with compact support. Since $f$ is invariant

Hence

$$
\int f H_{t} \phi=\int \phi H_{t}^{*} f=\int f \phi<\infty
$$

$$
\begin{aligned}
0 & =\frac{1}{t} \int_{E^{n}} f(a)\left(H_{t} \phi-\phi\right)(a) d a \\
& =\frac{1}{t} \int_{E^{n}} f(a) \int_{0}^{t}\left(H_{s}(G \phi)\right)(a) d s d a \\
& =\frac{1}{t} \int_{0}^{t} d s \int_{E^{n}} f(a)\left(H_{s}(G \phi)(a) d a\right. \\
& =\frac{1}{t} \int_{0}^{t} d s \int_{E^{n}} f(a) G \phi(a) d a \\
& =\int f(a) G \phi(a) d a=\int G^{*} f(a) \phi(a) d a .
\end{aligned}
$$

Since $\phi$ is arbitrary $G^{*} f=0$ provided that the interchange in the orders of integration is justified. But this is an obvious consequence of Fubini's theorem.

REMARK 7.3. Essentially the same arguments serve to establish an analogous theorem for nonnegative solutions of the equation $H_{t} f=f$.

We turn next to the proof of a converse to Theorem 7.2.

THEOREM 7.3. Suppose $f \in C^{2}\left(E^{n}\right)$ is a nonnegative solution of $G^{*} f=0$ and that $f(a)=\exp \left(O\left(\|a\|^{2}\right)\right)$ and $\partial f / \partial a_{n}=\exp \left(O\left(\|a\|^{2}\right)\right)$ as $\|a\| \rightarrow \infty$. Then $f$ is an invariant density for the process with generator $G$. 
Proof. The assumed restrictions on the growth of $f$ combined with Lemma 3.1 clearly imply the existence of a number $r>0$ such that $f(a) p(t ; a, b) \in L^{2}(d a)$ and $\left(\partial f / \partial a_{n}\right)(a) p(t ; a, b) \in L^{1}(d a)$ for $0<t \leqq r$. Furthermore by a routine application of the mean value theorem

$$
[p(t+h ; a, b)-p(t ; a, b)] / h=p(s ; a, b) O\left(\|a\|^{2}\right) \text { as }\|a\| \rightarrow \infty
$$

for some $s$ between $t$ and $t+h$.

Hence we can apply the dominated convergence theorem to conclude that

$$
\begin{aligned}
\frac{\partial}{\partial t} \int f p(t ; \cdot, b) & =\int f \frac{\partial}{\partial t} p(t ; \cdot, b) \\
& =\int f G p(t ; \cdot, b) \\
& =\int G^{*} f p(t ; \cdot, b)=0
\end{aligned}
$$

for $t<r$.

The restrictions on the growth of $f$ and $\partial f / \partial a_{n}$ are adequate to insure that no "boundary" terms appear in the transformation of $G$ acting on $p(t ; \cdot, b)$ into $G^{*}$ acting on $f$. We conclude that there exists a function $g$ which satisfies the equation

$$
\int f(a) p(t ; a, b) d a=g(b)
$$

for every $b \in E^{n}$ and every $0<t<r$.

Now let $\varepsilon>0$ be given. Choosing first a sphere of suitably small radius $\delta$ about $b$ and then applying formula (7.1) and the estimates furnished by Lemma 3.1 we can find a number $\gamma>0$ such that for all $0<t \leqq \gamma$

$$
\begin{aligned}
|g(b)-f(b)| \leqq & \left|g(b)-f(b) \int p(t ; a, b) d a\right|+\left|f(b)\left(1-\int p(t ; a, b) d a\right)\right| \\
\leqq & \int_{\|b-a\|<\delta}|f(a)-f(b)| p(t ; a, b) d a \\
& +f(b) \int_{\|b-a\| \geqq \delta} p(t ; a, b) d a+\int_{\|b-a\| \geqq \delta} f(a) p(t ; a, b) d a+\varepsilon \\
\leqq & \varepsilon+\varepsilon+\varepsilon+\varepsilon .
\end{aligned}
$$

(The assumed restriction on the rate of growth of $f$ enters into the estimate of the third integral.) Hence $g(b)=f(b)$ and thus

$$
\int f(a) p(t ; a, b) d a=f(b) \text { for } 0<t<r .
$$


Applying the Chapman-Kolmogoroff equation to both sides we see that in fact equality must hold for all $t>0$ and hence $f$ is an invariant density.

COROLlary 1. If $f(u)=\exp \left(-\sum C_{i j} u_{i} u_{j}-\sum B_{j} u_{j}\right)$ where $C_{i j}=C_{j i}$ and the $C_{i j}$ and the $B_{j}, i, j=1, \cdots, n$, satisfy the system of equations

$$
\begin{gathered}
C_{n j}\left(C_{n i}+a_{n+1-i}\right)+C_{n i}\left(C_{n j}+a_{n+1-j}\right)+C_{i-1, j}+C_{i, j-1}=0, \\
B_{n}\left(2 C_{n i}+a_{n+1-i}\right)+B_{i-1}=0, \\
-C_{n n}+B_{n}^{2} / 2-a_{1}=0, \quad i, j=1, \cdots, n
\end{gathered}
$$

with $B_{0}=C_{0, i}=C_{i, 0}=0$ then $f$ is an invariant density.

Proof. A routine computation shows that $G^{*} f=0$ if and only if the system of equations (7.2) is satisfied. Clearly then $f$ satisfies the hypothesis of Theorem 7.3.

It is useful to note that if $f=e^{-\phi}$ then $G^{*} f=0$ if and only if $\mathscr{D}^{*} \phi=a_{1}$ where

$$
\mathscr{D}^{*} \phi=\left(\frac{1}{2}\right)\left(\frac{\partial \phi}{\partial u_{n}}\right)^{2}-\left(\frac{1}{2}\right) \frac{\partial^{2} \phi}{\partial u_{n}^{2}}+\sum_{i=1}^{n-1} u_{i+1} \frac{\partial \phi}{\partial u_{i}}+\left(a_{1} u_{n}+\cdots+a_{n} u_{1}\right) \frac{\partial \phi}{\partial u_{n}} .
$$

Corollary 1 implies that every solution of (7.2) leads to an invariant density. If in particular $\operatorname{sp}(A) \subset \Lambda$ then we know by Theorem 7.1 that there can be only one solution to (7.2) namely $B_{i}=0$ and

$$
C_{i j}=\lim _{t \rightarrow \infty} R_{i j}^{-1}(t) / 2, \quad i, j=1, \cdots, n .
$$

Furthermore, by the same theorem $C_{i j}=0$ if $i+j \equiv 1(\bmod 2)$. When this last condition is imposed the system of equations (7.2) reduces to a more tractable form. We can then in fact show

THEOREM 7.4. Let $\phi(u)=\Sigma C_{i j} u_{i} u_{j}+\sum B_{j} u_{j}$ with $C_{i j}=C_{j i}$. Then under the constraint

$$
C_{i j}=0 \text { if } i+j \equiv 1(\bmod 2)
$$

the differential equation

$$
\mathscr{D}^{*} \phi=a_{1}
$$

has at most two solutions according as

$$
C_{n n-2 k}=0
$$

or

$$
C_{n n-2 k}=-a_{2 k+1}, \quad k=0,1, \cdots,[(n-1) / 2] .
$$

If either (1) $\operatorname{sp}(A) \subset \bar{\Lambda}$ or (2) $a_{1}<0$ or (3) both $a_{n}$ and $a_{1}$ differ from zero, then only solution (7.6) is possible. 
Proof. When $(i+j) \equiv 1(\bmod 2)$ then under the constraint $(7.3)$ the system of equations (7.2.1) reduces to

where

$$
C_{i-1, j}+C_{i, j-1}+C_{n i} a_{n+1-j}^{\prime \prime}+C_{n i} a_{n+1-i}^{\prime \prime}=0
$$

$$
\begin{aligned}
& a_{i}^{\prime \prime}=a_{i} \text { if } i \equiv 0(\bmod 2), \\
& a_{i}^{\prime \prime}=0 \text { if } i \equiv 1(\bmod 2) .
\end{aligned}
$$

But this implies that

$$
C_{i j}=-C_{i-1, j+1}-C_{n i} a_{n-j}^{\prime \prime}-C_{n j+1} a_{n+1-i}^{\prime \prime} \text { if } i+j \equiv 0(\bmod 2)
$$

so $C_{i j}$ is completely determined for $1 \leqq i, j \leqq n-1$ in terms of $C_{n n-2 k}$ and $a_{2 i}$, $k=0,1, \cdots,[(n-1) / 2], i=1,2, \cdots,[n / 2]$.

When $i+j \equiv 0(\bmod 2)$ then (7.2.1) and (7.3) together yield the system of equations

$$
C_{n j}\left(C_{n i}+a_{n+1-i}\right)+C_{n i}\left(C_{n j}+a_{n+1-j}\right)=0
$$

which immediately reduces to

$$
\begin{aligned}
C_{n n-2 k}\left(C_{n n-2 j}+a_{2 j+1}\right)+C_{n n-2 j}\left(C_{i n-2 k}+a_{2 k+1}\right) & =0, \\
k, j & =0,1, \cdots,[(n-1) / 2] .
\end{aligned}
$$

The system (7.8) has (at most) the two sets of solutions (7.5) and (7.6). If solution (7.6) holds then, by (7.2.2) and (7.2.3), $B_{i}=0, i=1, \cdots, n$. On the other hand if the sclution (7.5) holds then (7.2.2) and (7.2.3) imply in particular that

$$
\begin{aligned}
& B_{n} a_{n}=0, \\
& B_{n}^{2} / 2=a_{1} .
\end{aligned}
$$

Clearly if $a_{1}<0$ then (7.10) cannot hold so solution (7.5) is ruled out. If $a_{1}=0$ and $\operatorname{sp}(A) \subset \bar{\Lambda}$ then it is not hard to show that $a_{2 k+1}=0, k=0,1, \cdots,[(n-1) / 2]$ so that solutions (7.5) and (7.6) are the same. If $a_{1} \neq 0$ and $a_{n} \neq 0,(7.9)$ and (7.10) lead to a contradiction so solution (7.5) is impossible. This establishes the theorem.

Corollary 1. If $\phi=\Sigma C_{i j} u_{i} u_{j}$ with $C_{i j}=C_{j i}$ then under the constraint (7.3) the differential equation (7.4) has at most the two solutions corresponding to (7.5) and (7.6). If either $a_{1}=0$ or $\operatorname{sp}(A) \subset \bar{\Lambda}$ the solution (7.6) is unique.

Denote the particular invariant density which corresponds to (7.6) by $\psi_{0}$. Combining (7.6) with (7.7) it is easy to show

Corollary 2. Let $a_{0}=-1$. Then the invariant density

$$
\psi_{0}(u)=\exp \left(-\Sigma C_{i j} u_{i} u_{j}\right)
$$

is determined by the formulae 


$$
\begin{gathered}
C_{i j}=a_{n+1-i} a_{n-j}-C_{i-1, j+1} \text { if } i+j \equiv 0(\bmod 2), \\
C_{i j}=0 \text { if } i+j \equiv 1(\bmod 2) .
\end{gathered}
$$

It is perhaps mos efficient to compute $C_{i j}$ along each of the diagonals

$$
\{(i, j): i+j=2 k\}
$$

taking in turn $k=1, \cdots, n$ and proceeding from the upper right to the lower left (i.e. compute in the following order: $C_{11} ; C_{13}, C_{22} ; C_{15}, C_{24}, C_{33} ; \cdots$ ).

REMARK 7.4. Some restrictions on the $a_{i}, i=1, \cdots, n$, are necessary in order to insure a unique solution to the system of equations (7.2) even when the condition (7.3) is imposed. There are for example several stationary densities for the trajectory process arising from the differential operator $D\left(D-\lambda_{1}\right)\left(D-\lambda_{2}\right)$ $=D^{3}-a_{1} D^{2}-a_{2} D$ with $\lambda_{1}>0$, and $\lambda_{2}>0$. Routine computations show that for every solution of (7.2) $C_{11}=C_{21}=C_{31}=0$ but that the remaining coefficients may assume different sets of values. Under the restriction (7.3) there are three solutions

(1) $B_{1}=B_{2}=B_{3}=C_{32}=0, C_{33}=-a_{1}, C_{22}=a_{1} a_{2}$,

(2) and (3) $C_{32}=C_{33}=C_{22}=0, B_{3}= \pm\left(2 a_{1}\right)^{1 / 2}$,

$$
B_{2}=\mp a_{1}\left(2 a_{1}\right)^{1 / 2}, B_{1}=\mp a_{2}\left(2 a_{1}\right)^{1 / 2} \text {. }
$$

If the restriction (7.3) is lifted then there are four more solutions

(4) and (5) $\quad C_{32}=-a_{2}, C_{33}=-\lambda_{1}, C_{22}=-\lambda_{1} \lambda_{2}^{2}$,

$$
B_{3}= \pm\left(2 \lambda_{2}\right)^{1 / 2}, B_{2}=\mp\left(\lambda_{2}-\lambda_{1}\right)\left(2 \lambda_{2}\right)^{1 / 2}, B_{1}=\mp \lambda_{1} \lambda_{2}\left(2 \lambda_{2}\right)^{1 / 2} \text {, }
$$

(6) and (7) $C_{32}=-a_{2}, C_{33}=-\lambda_{2}, C_{22}=-\lambda_{1}^{2} \lambda_{2}, B_{3}= \pm\left(2 \lambda_{1}\right)^{1 / 2}$,

$$
B_{2}=\mp\left(\lambda_{1}-\lambda_{2}\right)\left(2 \lambda_{1}\right)^{1 / 2}, B_{1}=\mp \lambda_{1} \lambda_{2}\left(2 \lambda_{1}\right)^{1 / 2} \text {. }
$$

In the remainder of this paper we shall concern ourselves primarily with the particular invariant density $\psi_{0}$. If $\operatorname{sp}(A)$ is type I then we know (Theorem 9.2) that $\psi_{0}$ is, up to a multiplicative constant, the only invariant density. We suspect that in fact $\psi_{0}$ is unique whenever $\operatorname{sp}(A) \subset \bar{\Lambda}$ but lack proof. We shall show, nevertheless, in subsequent sections that $\psi_{0}$ may be uniquely characterized in an interesting fashion.

We conclude this section with

THEOREM 7.5. If $\psi_{0}(b)=\exp -\langle b, Q b\rangle$ then the following are equivalent

(1) $\operatorname{sp}(A) \subset \Lambda$,

(2) $Q$ is positive definite,

(3) $\psi_{0} \in L^{1}(d b)$.

Proof. If $\operatorname{sp}(A) \subset \Lambda$ then $|R(\infty)|<\infty$ so $2 Q=R^{-1}(\infty)$ is positive definite and consequently $\psi_{0}$ is integrable; so (1) implies (2) implies (3). Now suppose $\psi_{0}$ is integrable. If $\operatorname{sp}(A) \notin \Lambda$ then, as $t \rightarrow \infty,|R(t)| \rightarrow \infty$ and

$$
p(t ; b, c) \leqq|R(t)|^{-1 / 2} \rightarrow 0 .
$$


Hence we can apply the dominated convergence theorem to conclude that

$$
\psi_{0}(b)=\lim _{t \rightarrow \infty} \int \psi_{0} p(t ; \cdot, b)=\int \psi_{0} \lim _{t \rightarrow \infty} p(t ; \cdot, b)=0 .
$$

But this is not true, so (3) must imply (1).

This theorem leads immediately to a curious alternative to the Routh-Hurwitz criterion (see e.g. Gantmacher [10, p. 231]).

COROLlary 1. The polynomial $x^{n}+b_{1} x^{n-1}+\cdots+b_{n}$ with real coefficients is a Hurwitz polynomial, i.e. all its roots lie in $\Lambda$, if and only if the symmetric matrix $B$ is positive definite where (by formula 7.11 with $b_{t}=-a_{l}, i=0, \cdots, n$ )

$$
\begin{gathered}
B_{i j}=b_{n+1-i} b_{n-j}-B_{i-1, j+1} \quad \text { if } i+j \equiv 0(\bmod 2) \\
B_{i j}=0 \text { if } i+j \equiv 1(\bmod 2) \\
b_{0}=1, B_{0, i}=B_{n+1, i}=0, i, j=1, \cdots, n .
\end{gathered}
$$

Remark 7.5. Let $\psi_{0}\left(u_{1}, \cdots, u_{n}\right)=\sum_{i, j=1}^{n} Q_{i j} u_{i} u_{i}$ be the special invariant density for the trajectory process generated by $P(D)$. Then $\psi_{0}\left(u_{1}, \cdots, u_{n+2}\right)$ $=\sum_{i, j=1}^{n} Q_{i j}\left(u_{i+2}+\beta^{2} u_{i}\right)\left(u_{j+2}+\beta^{2} u_{j}\right)$ is the special invariant density which corresponds to $\left(D^{2}+\beta^{2}\right) P(D)$ and $\psi_{0}\left(u_{1}, \cdots, u_{n+1}\right)=\sum_{i, j=1}^{n} Q_{i j} u_{i+1} u_{j+1}$ is the special invariant density corresponding to $D P(D)$.

8. A characterization of the special invariant density $\psi_{0}$.

THEOREM 8.2. Consider the trajectory process generated by the differential operator $P(D)=D^{n}-a_{1} D^{n-1}-\cdots-a_{n}$. Then $-\log \psi_{0}$ may be uniquely characterized as the form $\eta(u)=\Sigma C_{i j} u_{i} u_{j}+\Sigma B_{j} u_{j}, C_{i j}=C_{j l}$, which

(1) is invariant on the streamlines of the "force free oscillator" $\left(D-a_{2} D^{n-2}-a_{4} D^{n-4}-\cdots\right) v_{1}=0$ and for which

(2) $C_{n n-2 k}=-a_{2 k+1}, k=0,1, \cdots,[(n-1) / 2]$.

We first prove

THEOREM 8.1. Let $\phi(u)=\Sigma C_{i j} u_{i} u_{j}, C_{i j}=C_{j i}$. Then the conditions

$$
\begin{gathered}
\mathscr{D}^{*} \phi=a_{1}, \\
C_{i j}=0 \text { if } i+j \equiv 1(\bmod 2)
\end{gathered}
$$

are equivalent to the conditions

$$
\begin{aligned}
& \mathscr{D}_{1} \phi=a_{1}, \\
& \mathscr{D}_{2} \phi=0,
\end{aligned}
$$

where 


$$
\begin{aligned}
& \mathscr{D}_{1} \phi=\frac{1}{2}\left(\frac{\partial \phi}{\partial u_{n}}\right)^{2}-\frac{1}{2}\left(\frac{\partial^{2} \phi}{\partial u_{n}^{2}}\right)+\left(a_{1} u_{n}+a_{3} u_{n-2}+\cdots\right) \frac{\partial \phi}{\partial u_{n}} \\
& \mathscr{D}_{2} \phi=\mathscr{D}^{*} \phi-\mathscr{D}_{1} \phi=\sum_{i=1}^{n-1} u_{i+1} \frac{\partial \phi}{\partial u_{i}}+\left(a_{2} u_{n-1}+a_{4} u_{n-3}+\cdots\right) \frac{\partial \phi}{\partial u_{n}} .
\end{aligned}
$$

Proof. $\mathscr{D}_{2} \phi=0$ if and only if for all $1 \leqq i, j \leqq n$,

$$
C_{i-1, j}+C_{i, j-1}+C_{n i} a_{n+1-j}^{\prime \prime}+C_{n j} a_{n+1-i}^{\prime \prime}=0
$$

where

$$
\begin{aligned}
& a_{j}^{\prime \prime}=a_{j} \text { if } j \equiv 0(\bmod 2), \\
& a_{j}^{\prime \prime}=0 \text { if } j \equiv 1(\bmod 2) .
\end{aligned}
$$

Or equivalently if and only if

$$
C_{i j}=-C_{i-1, j+1}-C_{n i} a_{n-j}^{\prime \prime}-C_{n j+1} a_{n+1-i}^{\prime \prime} .
$$

If $j+i \equiv 0(\bmod 2)$ then $(8.3)$ coincides with (7.7). If $i+j \equiv 1(\bmod 2)$ then (8.3) reduces to

$$
C_{i j}=-C_{i-1, j+1}
$$

which implies that $C_{i j}=0$ whenever $i+j \equiv 1(\bmod 2)$.

On the other hand $\mathscr{D}_{1} \phi=a_{1}$ if and only if

$$
\begin{array}{lc}
\text { (8.4.1) } & C_{n j}\left(C_{n i}+a_{n+1-i}^{\prime}\right)+C_{n i}\left(C_{n j}+a_{n+1-j}^{\prime}\right)=0, \\
\text { (8.4.2) } & C_{n n}+a_{1}=0 \\
\text { where } & a_{i}^{\prime}=a_{i} \quad \text { if } i \equiv 1(\bmod 2), \\
& a_{i}^{\prime}=0 \quad \text { if } i \equiv 0(\bmod 2) .
\end{array}
$$

Under the restriction $C_{i j}=0$ for $i-j \equiv 1(\bmod 2)$, which must hold if $\mathscr{D}_{2} \phi=0,(8.4 .1)$ reduces to (7.8) and the theorem follows.

REMARK 8.1. If $\phi(u)=\Sigma C_{i j} u_{i} u_{j}$ with $C_{i j}=C_{j l}$ then (8.1) and (8.2) together have at most the two solutions: (1) $\phi=0$, (2) $\phi=-\log \psi_{0}$. If either $a_{1} \neq 0$ or $\operatorname{sp}(A) \subset \bar{\Lambda}$ then only solution (2) is possible.

REMARK 8.2. Theorem 8.1 is not valid, without further restrictions, when applied to the more general form $\eta(u)=\Sigma C_{i j} u_{i} u_{j}+\Sigma B_{j} u_{j}, C_{i j}=C_{j i}$. For routine computations show that if $\mathscr{D}_{1} \eta=a_{1}$ and $\mathscr{D}_{2} \eta=0$ then $B_{j}=0, j=1, \cdots, n$. Whereas there exist cases where $\eta$ is a solution of (7.3) and (7.4) and some of the $B_{i}$ are nonzero. (See e.g. the case discussed in Remark 7.4.) If care is taken to circumvent these cases by, for example, restricting $\operatorname{sp}(A) \subset \bar{\Lambda}$ then Theorem 8.1 may be extended to this more general class of forms.

Analyzing the role played by each of the conditions (8.1) and (8.2) in the proof of Theorem 8.1 it is readily seen that $-\log \psi_{0}$ may be characterized as the unique form, $\eta(u)=\Sigma C_{i j} u_{i} u_{j}+\Sigma B_{j} u_{j}, C_{i j}=C_{j i}$, which satisfies 
(1) $C_{n n-2 k}=-a_{2 k+1}, k=0,1, \cdots,[(n-1) / 2]$.

(2) $\mathscr{D}_{2} \eta=0$.

A trajectory (or streamline) in phase space $E^{n}$ of the "oscillator"

$$
\left(D^{n}-a_{2} D^{n-2}-a_{4} D^{n-4}-\cdots\right) v_{1}=0 \quad(t \geqq 0)
$$

is a curve $v(t)=\left(v_{1}(t), \cdots, v_{n}(t)\right)$ for which

$$
\begin{aligned}
& \dot{v}_{i}=v_{i+1}, \quad i=1, \cdots, n-1, \\
& \dot{v}_{n}=a_{2} v_{n-1}+a_{4} v_{n-3}+\cdots .
\end{aligned}
$$

A differentiable function $g$ on $E^{n}$ is said to be invariant under the streaming of this "oscillator" (or on the streamlines of this "oscillator") if $g$ is constant on each such curve. That is, if

$$
\begin{aligned}
\frac{d}{d t} g \circ v & =\sum_{i=1}^{n} \frac{\partial g}{\partial v_{i}} \frac{d v_{i}}{d t} \\
& =\sum_{i=1}^{n-1} v_{i+1} \frac{\partial g}{\partial v_{i}}+\left(a_{2} v_{n-1}+a_{4} v_{n-3}+\cdots\right) \frac{\partial g}{\partial v_{n}}=0 .
\end{aligned}
$$

This, however, is precisely the condition $\mathscr{D}_{2} g=0$. This observation serves to establish Theorem 8.2.

REMARK 8.3. If the roots of the polynomial $P(x)=x^{n}-a_{1} x^{n}-\cdots-a_{n}$ lie in $\Lambda$ then the roots of the polynomial $P_{e}(x)=x^{n}-a_{2} x^{n-2}-a_{4} x^{n-4}-\cdots$ have real parts equals to zero. (Gantmacher [1, p. 271]). Hence it is reasonable, at least in this case, to refer to the "force free oscillator" $P_{e}(D) v_{1}=0$ as the associated "undamped oscillator".

REMARK 8.4. Let $\phi(u)=-\log \psi_{0}(u)=\langle u, Q u\rangle$. Since, for example, $\partial \phi / \partial a_{1}=\phi$ with $a_{1}=1, a_{3}=a_{5}=\cdots=0$ and $\mathscr{D}_{2} \phi=0$ regardless of the values chosen for $a_{1}, a_{3}, \cdots$ it follows that the function $\partial \phi / \partial a_{1}$ is invariant under the streaming of the "undamped oscillator". For analogous reasons $\partial \phi / \partial a_{3}, \partial \phi / \partial a_{5}, \cdots$ are also invariant under the streaming of the "undamped oscillator" and in fact the collection of all such functions forms a basis for $\phi$ :

$$
\phi=a_{1} \frac{\partial \phi}{\partial a_{1}}+a_{3} \frac{\partial \phi}{\partial a_{3}}+\cdots .
$$

9. The backward process; a second characterization of $\psi_{0}$. Suppose $\psi$ is an invariant density for the process $y$. Let $P_{\psi}$ denote the unique extension for the smallest Borel field containing the cylinder sets of the measure which assigns to such a set, say

$$
\left[\omega \in \Omega: \omega\left(t_{1}\right) \in B_{1}, \cdots, \omega\left(t_{n}\right) \in B_{n}\right], \quad 0 \leqq t_{1}<\cdots<t_{n}
$$

a weighting

$$
\int_{B_{1}} \psi\left(a_{1}\right) d a_{1} \int_{B_{2}} p\left(t_{2}-t_{1} ; a_{1}, a_{2}\right) d a_{2} \cdots \int_{B_{n}} p\left(t_{n}-t_{n-1} ; a_{n-1}, a_{n}\right) d a_{n} ;
$$


the $B_{i}, i=1, \cdots, n$, are Borel sets in $E^{n}$. Note that since $\psi$ is invariant and $p$ satisfies the Chapman-Kolmogorov equation the prescription for assigning weights to cylinder sets is consistent (e.g. the same weight is assigned to $\left[\omega: \omega\left(t_{1}\right) \in E^{n}, \omega\left(t_{2}\right) \in B_{2}\right]$ as to $\left.\left[\omega: \omega\left(t_{2}\right) \in B_{2}\right]\right)$.

The invariance of $\psi$ also implies that $P_{\psi}$ is a stationary measure though in general it need not be finite. The process $y=\left([0, \infty) \times \Omega, E^{n},\left\{P_{a}: a \in E^{n}\right\}\right)$ thus has a natural extension to a "process" $\left((-\infty, \infty) \times W, E^{n}, P_{\psi}\right)$ where the new path space $W$ is the set of continuous functions from $(-\infty, \infty)$ to $E^{n}$; if $0 \leqq t_{1}<\cdots<t_{n}$ then

$$
\begin{aligned}
P_{\psi}\left(y\left(-t_{1}\right) \in B_{1}, \cdots, y\left(-t_{n}\right) \in B_{n}\right) & \\
& =P_{\psi}\left(y(0) \in B_{n}, y\left(t_{n}-t_{n-1}\right) \in B_{n-1}, \cdots, y\left(t_{::}-t_{1}\right) \in B_{1}\right) .
\end{aligned}
$$

We shall denote the extended "process" by $y$ also; the word process is enclosed in quotation marks to emphasize the fact that $P_{\psi}(W)$ may be infinite.

Now for $t>0$ let

$$
\hat{p}(t ; a, b)=p(t ; b, a) \psi(b) / \psi(a) .
$$

It is easily verified that $\hat{p}$ satisfies the Chapman-Kolmogorov equation, that $\hat{p}\left(t ; \cdot, E^{n}\right) \equiv 1$ on $E^{n}$ and that $\psi$ is invariant relative to $\hat{p}$.

Hence there exists a stationary Markov "process" $\hat{y}=\left((-\infty, \infty) \times W, E^{n}, \hat{P}_{\psi}\right)$ with transition probability density $\hat{p}(t ; a, b)$ where

$$
\begin{aligned}
\hat{P}_{\psi}[\omega & \left.\in W: \omega\left(t_{1}\right) \in B_{1}, \cdots, \omega\left(t_{n}\right) \in B_{n}\right] \\
& =\hat{P}_{\psi}\left(\hat{y}\left(t_{1}\right) \in B_{1}, \cdots, \hat{y}\left(t_{n}\right) \in B_{n}\right) \\
& =\int_{B_{1}} \psi\left(a_{1}\right) d a_{1} \int_{B_{2}} \hat{p}\left(t_{2}-t_{1} ; a_{1}, a_{2}\right) d a_{2} \cdots \int_{B_{n}} \hat{p}\left(t_{n}-t_{n-1} ; a_{n-1}, a_{n}\right) d a_{n} .
\end{aligned}
$$

In particular if $t_{2}-t_{1}=t>0$, then

$$
\begin{aligned}
\hat{P}_{\psi}\left(\hat{y}\left(t_{1}\right) \in d a, \hat{y}\left(t_{2}\right) \in d b\right) & =\psi(a) d a \hat{p}(t ; a, b) d b \\
& =\psi(b) d b p(t ; b, a) d a \\
& =P_{\psi}(y(0) \in d b, y(t) \in d a) \\
& =P_{\psi}(y(0) \in d a, y(-t) \in d b) \\
& =P_{\psi}\left(y\left(t_{1}\right) \in d b, y\left(t_{2}\right) \in d a\right) .
\end{aligned}
$$

Hence we may identify $\hat{y}(t)$ with $y(-t)$ and think correspondingly of $y$ as the "forward-process" and of $\hat{y}$ as the "backward-process".

The generator for the backward process, $\hat{G}$, is equal to the adjoint, relative to $\psi$, of the forward generator $G$. That is, if $f$ and $g$ are $C^{\infty}$ functions with compact support 


$$
\int(G f) g \psi=\int f(\hat{G} g) \psi
$$

In the case at hand

$$
\hat{G} g=\left(\frac{1}{2}\right) \frac{\partial^{2} g}{\partial u_{n}^{2}}+\sum_{i=1}^{n-1} u_{i+1} \frac{\partial g}{\partial u_{i}}-\left(a_{1} u_{n}+\cdots+a_{n} u_{1}-\frac{1}{\psi} \frac{\partial \psi}{\partial u_{n}}\right) \frac{\partial g}{\partial u_{n}}
$$

If in particular

$$
\psi=\exp \left(-\Sigma C_{i j} u_{i} u_{j}\right)-\Sigma H_{j} u_{j}
$$

then

$$
\frac{1}{\psi} \frac{\partial \psi}{\partial u_{n}}=-2 \sum C_{n j} u_{j}-H_{n}
$$

Consequently

$$
\hat{G} g=\frac{1}{2} \frac{\partial^{2} g}{\partial u_{n}^{2}}-\sum_{i=1}^{n-1} u_{i+1} \frac{\partial g}{\partial u_{i}}-\left[\sum_{j=1}^{n}\left(a_{n+1-j}+2 C_{n j}\right) u_{j}+H_{n}\right] \frac{\partial g}{\partial u_{n}} .
$$

The stochastic differentials for $\hat{y}$ may be deduced from the coefficients of $\hat{G}$ Ito $[11$, pp. 172-210]); i.e.

$$
\begin{aligned}
& d \hat{y}_{i}=-\hat{y}_{i+1} d t, i=1, \cdots, n-1, \\
& d \hat{y}_{n}=-\left[\sum_{j=1}^{n}\left(a_{n+1-j}+2 C_{n j}\right) \hat{y}_{j}+H_{n}\right] d t+d b_{n} .
\end{aligned}
$$

In other words $\hat{y}$ is the formal solution of the vector stochastic differential equation

$$
D \hat{y}(t, \omega)=\hat{A} \hat{y}(t, \omega)+\dot{b}(t, \omega)+h
$$

where

$$
\begin{aligned}
& \hat{y}=\left[\begin{array}{c}
\hat{y}_{1} \\
\vdots \\
\vdots \\
\hat{y}_{n}
\end{array}\right], \quad b=\left[\begin{array}{c}
0 \\
\vdots \\
0 \\
b_{n}
\end{array}\right], \quad h=\left[\begin{array}{r}
0 \\
\vdots \\
0 \\
-H_{n}
\end{array}\right] \text {, } \\
& \hat{A}=\left[\begin{array}{rrrrr}
0 & -1 & 0 & \cdot & 0 \\
0 & 0 & -1 & & 0 \\
& & \cdot & & -1 \\
-\hat{a}_{n} & \cdot & . & & -\hat{a}_{1}
\end{array}\right] \text {, }
\end{aligned}
$$


The spectrum of $\hat{A}$ is equal to the set of roots of the polynomial

$$
(-x)^{n}-\hat{a}_{1}(-x)^{n-1}-\cdots-\hat{a}_{n} .
$$

Recall that the spectrum of the corresponding matrix for the forward process, $A$, is equal to the set of roots of the polynomial $x^{n}-a_{1} x^{n-1}-\cdots-a_{n}$. A routine check then shows that $\operatorname{sp}(A)=\operatorname{sp}(\hat{A})$ if and only if

$$
\begin{aligned}
& C_{n n-j}=-a_{j+1} \quad \text { if } j \equiv 0(\bmod 2), \\
& C_{n n-j}=0 \text { if } j \equiv 1 \quad(\bmod 2), \quad j=0, \cdots, n-1 .
\end{aligned}
$$

It is also easily checked that the only solution to the system of equation (7.2) which satisfies the conditions (9.1) is the negative exponent of the special invariant density we have termed $\psi_{0}$. This establishes

THEOREM 9.1. $-\log \psi_{0}(u)$ may be uniquely characterized as the form $\Sigma C_{i j} u_{i} u_{j}+\Sigma H_{j} u_{j}, C_{i j}=C_{j i}$, relative to which the spectrum of the "forward" matrix $A$ is identical to the spectrum of the "backward" matrix $\hat{A}$.

Remark 9.1. Let $\hat{A}_{0}$ denote the matrix for the backward process relative to $\psi_{0}$. Then the matrix $U=\left(A-\hat{A}_{0}\right) / 2$ characterizes the undamped oscillator discussed in $\S 8$.

THEOREM 9.2. If the (forward) trajectory process $y$ is recurrent the invariant density $\psi_{0}$ is unique up to a multiplicative constant.

Proof. Let $f$ be an invariant density for the process $y$ and consider the backward process $\hat{y}$ relative to the invariant density $\psi_{0}$. The corresponding transition density $\hat{p}(t ; a, b)=p(t ; b, a) \psi_{0}(b) / \psi_{0}(a)$. If $\hat{H}_{t}$ denotes the corresponding transition operator then

$$
\left(H_{t} \frac{f}{\psi_{0}}\right)(a)=\int \hat{p}(t ; a, b) \frac{f(b)}{\psi_{0}(b)} d b=\frac{f(a)}{\psi_{0}(a)} .
$$

Thus for each $t>0$ the mean of the nonnegative random variable $n(t)$ $=f(\hat{y}(t)) / \psi_{0}(\hat{y}(t))$, conditioned on a starting point $a \in E^{n}$, is equal to

$$
\int_{E^{n}} \hat{p}(t ; a, b) n(b) d b=n(a) .
$$

Hence $n(t), t \in(0, \infty)$, is a continuous (positive) martingale, and therefore converges with probability one as $t \rightarrow \infty$. But this implies that $f / \psi_{0}$ must be a constant. For, if not, then there exist two disjoint open nonempty sets on which $f / \psi_{0}$ takes distinct values. However, since the forward process is recurrent the backward process is also recurrent and thus $\hat{y}(t)$ visits each of these two sets infinitely often, for arbitrarily large $t$, with probability one. This then implies that $n(t)$ cannot converge with probability one which is a contradiction. (For a discussion of the martingale convergence theorem see Doob [6, Theorem 4.1, p. 354]). 


\section{BIBLIOGRAPHY}

1. E. F. Beckenbach and R. Bellman, Inequalities, Springer-Verlag, Berlin, 1961.

2. G. Birkhoff and G. C. Rota, Ordinary differential equations, Ginn, New York, 1962.

3. S. Bochner and W. T. Martin, Several complex variables, Princeton Univ. Press, Princeton, N. J., 1948.

4. S. Chandrasekhar, Stochastic problems in physics and astronomy, Rev. Modern Phys. 15 (1943), 1-89. (Reprinted in [21].)

5. E. A. Coddington and N. Levinson, Theory of ordinary differential equations, McGrawHill, New York, 1955.

6. J. L. Doob, Stochastic processes, Wiley, New York, 1959.

7. - The Brownian movement and stochastic equations, Ann. of Math. 43 (1942), 351-369. (Reprinted in [21].)

8. —_ The elementary Gaussian process, Ann. Math. Statist. 15 (1944), 229-282.

9. E. B. Dynkin, Infinitesimal operators of Markov processes, Theor. Probabilility Appl. 1 (1956), 34-53.

10. F. R. Gantmacher, Applications of the theory of matrices, Interscience, New York, 1959.

11. K. Ito, Lectures on stochastic processes, Tata Institute of Fundamental Research, Bombay, 1961.

12. - The expected number of zeros of continuous stationary Gaussian process, $\mathbf{J}$. Math. Kyoto Univ. 3 (1964), 207-216.

13. M. Kac, Probability theory; its role and impact, SIAM Rev. 4 (1962), 1-11.

14. R. Z. Khasminskii, Ergodic properties of recurrent diffusion processes and stabilization of the solution to the Cauchy problem for parabolic equations, Theor. Probability Appl. 5 (1960), 179-196.

15. M. Loève, Probability theory, 2nd ed., Van Nostrand, Princeton, N. J., 1960.

16. H. P. McKean, Jr., $A$ winding problem for a resonator driven by a white noise, J. Math. Kyoto Univ. 2 (1963), 227-235.

17. G. Maruyama and H. Tanaka, Ergodic property of $\mathrm{N}$-dimensional recurrent Markov processes, Mem. Fac. Sci. Kyushu Univ. Ser. A 13 (1959), 157-172.

18. Ming Chen Wang and G. E. Uhlenbeck, On the theory of the Brownian motion, Rev. Modern Phys. 17 (1945), 323-342. (Reprinted in [21].)

19. E. Nelson, The adjoint Markoff process, Duke Math. J. 25 (1958), 671-690.

20. J. Potter, Some statistical properties of the motion of a non-linear oscillator driven by white noise, Ph.D. thesis, M.I.T., Cambridge, Mass.; Trans. Amer. Math. Soc. to appear).

21. N. Wax (editor), Selected papers on noise and stochastic processes, Dover, New York, 1954.

Massachusetts Institute of Technology,

CAMBridge, MassachusetTS 